Multiscale Restriction Smoothed Basis Method for Fractured Porous Media (F-MsRSB)

16 August, 2015

Swej Shah 
Title : Multiscale restriction smoothed basis method for fractured porous media

(F-MsRSB)

$\begin{array}{lll}\text { Author(s) } & : \text { Swej Shah } \\ \text { Date } & : \text { August 17, } 2015 \\ \text { Professor(s) } & : \text { Dr. Hadi Hajibeygi, Principal Advisor } \\ & \text { Prof. Dr. Knut-Andreas Lie, Co-Advisor } \\ \text { Supervisor(s) } & : \text { Ir. Matei Țene } \\ & \text { Mr. Olav Møyner } \\ \text { Postal Address } & : \text { Section for Petroleum Engineering and Geosciences } \\ & \text { Department of Geoscience and Engineering } \\ & \text { Delft University of Technology } \\ & \text { P.O. Box } 5028 \\ \text { Telephone } & : \text { The Netherlands } \\ \text { Telefax } & : \text { (31) } 152781328 \text { (secretary) } 152781189\end{array}$

Copyright (C) 2015, Section for Petroleum Engineering and Geosciences All rights reserved. No parts of this publication may be reproduced, stored in a retrieval system, or transmitted, In any form or by any means, electronic, mechanical, photocopying, recording, or otherwise, without the prior written permission of the Section for Petroleum Engineering and Geosciences, TU Delft. 


\title{
TंUDelft
}

Delft University of Technology

Faculty of Civil Engineering and Geosciences

Department of Geoscience \& Engineering

\section{Multiscale Restriction Smoothed Basis Method for Fractured Porous Media (F-MsRSB)}

\author{
Thesis submitted to the \\ Delft University of technology \\ in partial fulfilment of the requirements \\ for the degree \\ MASTER OF SCIENCE \\ IN \\ APPLIED EARTH SCIENCES
}

by

\author{
Swej Shah \\ S.Y.Shah@student.tudelft.nl
}

Delft, the Netherlands

August 17, 2015

Copyright (c) 2015 Swej Shah. All rights reserved.

An electronic version of this dissertation is available at http://repository.tudelft.nl/. 


\section{Author}

Swej Shah

MSc Student

S.Y.Shah@student.tudelft.nl

Track: Petroleum Engineering and Geosciences

Specialization: Petroleum Engineering

Department of Geoscience \& Engineering

Delft University of Technology

\section{Title}

Multiscale Restriction Smoothed Basis Method for Fractured Porous Media (F-MsRSB)

\section{Committee Members}

Dr. Hadi Hajibeygi

H.Hajibeygi@tudelft.nl

Prof. Dr. Knut-Andreas Lie

Knut-Andreas.Lie@sintef.no

Prof. Dr. Ir. Kees Vuik

C.Vuik@tudelft.nl

MSc. Ir. Matei Ţ Tene

M.Tene@tudelft.nl
Principal Advisor

Assistant Professor

Department of Geoscience \& Engineering

Delft University of Technology

Co-Advisor

Chief Scientist

Department of Applied Mathematics

Sintef ICT

Professor of Numerical Mathematics

Department of Applied Mathematics

Delft University of Technology

PhD Candidate

Department of Geoscience \& Engineering

Delft University of Technology 


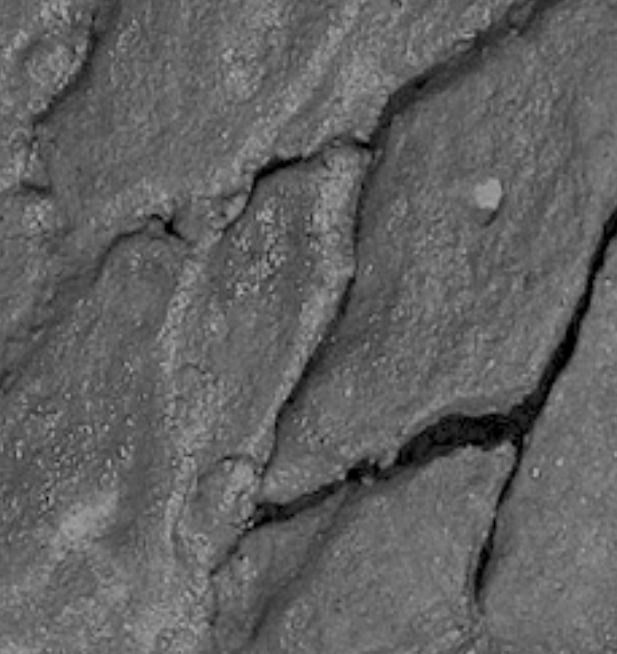

\section{Multiscale restriction smoothed basis} method for fractured porous media F-MsRSB

\section{Master Thesis - Applied Earth Sciences}

Swej Shah

swej.shah@outlook.com August 17, 2015 
This thesis is dedicated to my parents, Neela and Yogesh Shah, for their love, endless support and encouragement. 
"There are no facts, only interpretations." -Friedrich Nietzsche 


\begin{abstract}
A novel multiscale method for multiphase flow in heterogeneous fractured porous media (F-MsRSB) is devised. The discrete fine-scale system is governed on the basis of embedded fracture modelling approach, which benefits from independent grids for heterogeneous rock (matrix) and lower-dimensional high-flow-rate fracture domains. Given this fine-scale discrete system, following the algebraic multiscale method for fractured media (F-AMS) [1,2], F-MsRSB imposes independent coarse grids on matrix and fracture fine-scale cells. Matrix and fracture basis functions are then solved following the restriction smoothed procedure (MsRSB) [3], allowing for more flexibility and improved robustness in treatment of complex geometrical features and heterogeneous coefficients. These basis functions construct the prolongation operator which maps between the coarse- and fine-scale systems. Similar to F-AMS, F-MsRSB allows for general coupling of matrix and fracture basis functions, thus efficient for treatment of large variety of fracture conductivities. In addition, it has the advantage of adaptive global smoothing strategies to update the basis functions, as in the original MsRSB for non-fractured media. F-MsRSB is conservative, described and implemented in an algebraic form. More important, employing it to unstructured and rectilinear grids for fractured media is straightforward. Through several challenging test cases for single and multiphase flow, using realistic and synthetic fracture maps along with heterogeneous matrix, it is concluded that F-MsRSB is quite efficient and accurate for heterogeneous fractured media. Benefiting from the two recent multiscale developments, F-AMS and MsRSB, F-MsRSB casts a new approach to treat complex large-scale fractured porous media. The work of this thesis will be made available in the public domain as a module for the open-source MATLAB Reservoir Simulation Toolbox (MRST) developed by SINTEF Applied Mathematics.
\end{abstract}




\section{Acknowledgments}

This thesis marks the end of my academic journey and the start of new beginnings. The freedom and independence allowed while researching this challenging topic has left me in contentment, with a feeling of accomplishment.

The successful realization of this endeavour would never have been possible without the guidance of Dr. Hadi Hajibeygi, my principal advisor. Hadi's patience, motivation and immense knowledge was crucial towards both personal and professional development I underwent in the two productive years I spent at TU Delft. I don't think any other supervisor would have been as involved with my day to day work as Hadi was and because of the several Skype calls we had, I seldom strayed from the primary target of my master thesis. A heartfelt thanks to Prof. Knut-Andreas Lie, my co-advisor, for many insightful talks and encouraging remarks during my time at Sintef in Oslo. He once told me jokingly, "The only reward for hard work is the expectancy of more hard work, and before you realise, you will get used to it." Without his acquaintance and timely advice, I may have failed to look at the big picture, of which the work we do is just a small part. Hadi and Knut-Andreas gave me the opportunity of working with the talented Applied Mathematics team at Sintef in a very friendly atmosphere and for that I will always be grateful.

A special thank you to Olav Møyner and Matei Țene for the numerous times I walked up to your desk, often puzzled with questions, but always left with valuable inputs and a clear mind. To put it into perspective, Olav and Matei are the reason why I did not lose my mind when things weren't working they way I had imagined. Sindre Hilden, my office-mate and a good friend introduced me to the delicious Norwegian "boller". I would like to thank him for our frequent deep and worldly conversations in the middle of the day, and numerous coffee-breaks which helped alleviate the monotony and lighten up the mood.

Ayush, Sander, Meeneesh and Robin were always attentive through my relatively long presentations at the DARSim group meetings. Their comments were honest and key towards improving not only my presentation skills but also the overall quality of my research. I also thank the Chevron/Schlumberger Intersect Alliance for sponsoring this work.

Then there are several others including my parents and my sister, who I cannot thank enough. My friends ensured that I had a functional social life. They always distracted me from my thesis when necessary. All of them have played an integral role in helping me accomplish my goals, for which I will always be ever so grateful. 


\section{Contents}

1 Introduction 1

2 Governing equations and fine-scale system 5

3 Multiscale Restriction Smoothed Basis Method (MsRSB)

3.1 Algebraic multiscale formulation . . . . . . . . . . . . . 7

3.2 Coarse grid and interaction regions . . . . . . . . . . . . . . . . 8

3.3 MsRSB prolongation operator . . . . . . . . . . . . . . . 9

4 MsRSB for Fractured Media (F-MsRSB) 14

4.1 Algebraic formulation . . . . . . . . . . . . . . . . . . 14

4.2 Interaction regions and basis functions . . . . . . . . . . . 15

5 Numerical Results $\quad 18$

5.1 Sensitivity to Coarse DOF in fracture . . . . . . . . . . . . . 18

5.2 F-MsRSB for heterogeneous fractured media . . . . . . . . . . . . . 19

5.3 3D models . . . . . . . . . . . . . . . . . . . . 23

6 Conclusion $\quad 31$

$\begin{array}{ll}\text { References } & 32\end{array}$ 


\section{List of Figures}

1.1 Outcrop images of naturally fractured rocks . . . . . . . . . . 2

3.1 Highlighted cells in turquoise colour are the interaction region for a coarse node (a and $\mathbf{c}$ ). Also shown in yellow, in the same plots (a and c), are the interaction boundary cells. Shown on the right are the global boundary cells $G$, highlighted in yellow (b and $\mathbf{d}$ ). The top row shows a rectangular grid with a uniform coarse partition, while the bottom row presents a hexagonal grid with an unstructured coarse partition. . . . .

3.2 Restriction smoothed basis function computed iteratively over a $100 \mathrm{~m}$ long 1D homogeneous domain with $20 \mathrm{~m}$ coarse blocks. Vertical axis gives the basis function value for the $3^{\text {rd }}$ coarse block. Tolerance for convergence $=10^{-3}$

3.3 Restriction smoothed basis function for coarse block $(2,2)$ computed iteratively over a $60 \times 60$ homogeneous domain with $3 \times 3$ coarse blocks. Tolerance for convergence $=10^{-3} \ldots \ldots \ldots \ldots$

3.4 Illustration of the restriction smoothed basis functions (d-f) and classical MSFV basis functions (g-i) for three different permeability fields: homogeneous (a), heterogeneous patchy-field (b) and heterogeneous chan-

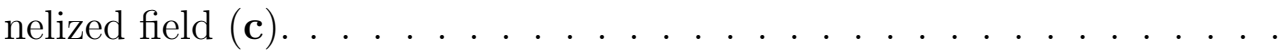

4.1 Illustration of multiscale grids for matrix and fracture (a) with interaction regions for matrix (b) and fracture (c) for a case with $30 \times 30$ matrix and 20 fracture fine-scale cells. Multiscale coarse grid contains $3 \times 3$ matrix blocks and 2 blocks inside the fracture. Here, $a=1 / 25$ and, consequently, $d=7 \ldots \ldots \ldots \ldots$

4.2 Matrix and fracture basis functions for the system described in Fig. 4.117

5.1 First test case which contains $100 \times 100$ matrix and 200 fracture cells at fine scale, with homogeneous $2 \mathrm{D}$ rock formation. The left and right boundaries are subject to Dirichlet values of 1 and 0 , respectively. . . .

5.2 F-MsRSB coarse grids for matrix and fractures. The matrix coarse grid consists of $10 \times 10$ fine cells, while the fracture coarse cells are varied from 1 (200 fine cells in 1 block) to 5 (40 cells in each block) and 10 (20 cells in each block). . . . . . . . . . . . . . . . . . . .

5.3 Reference and F-MsRSB pressure for the first test case as shown in Fig. 5.1. F-MsRSB solutions are presented for different fracture coarsening ratios. . . . . . . . . . . . . . . . . . 20 20

5.4 Convergence of F-MsRSB+ILU(0) for different DOF in fracture. . . . . 21 
5.5 Matrix coarse grid showing well locations and fracture map extracted from an outcrop (a); Logarithm of the corresponding permeability field in the matrix (b).

5.6 Reference saturation profile compared with saturation maps obtained after one F-MsRSB cycle at different PV injected. Absolute errors in saturation are also shown in (g)-(i).

5.7 Saturation at producer grid-block (a) and global saturation error (b) for outcrop model as a function of simulation time measured in porevolume-injection. Shown are the corresponding results for non-iterative F-MsRSB and iterative F-MsRSB with the tolerances of 0.1 and 0.01 on the pressure solves. . . . . . . . . . . . . . . . .

5.8 Matrix coarse grid with 100 DOF, fracture map and well locations (a); Petrophysical rock properties sampled from the $10^{\text {th }}$ layer of the tarbert

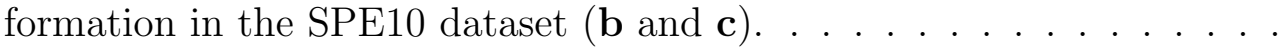

5.9 Saturation maps for fine-scale reference and F-MsRSB after 1 cycle of iteration (F-MsRSB + ILU $(0))$ at different simulation times. . . . . . .

5.10 Production and injection quantities at well locations for the statistical 2D fracture map. . . . . . . . . . . . . . . . . .

5.11 Illustration of the first 3D case, with a fine-scale grid that contains $50 \times 50 \times 50$ matrix cells and two intersecting fracture planes that each contain $100 \times 30$ fracture cells. Also shown on the right is heterogeneous matrix permeability map, along with the imposed $20 \times 20 \times 20$ coarse grid used by F-MsRSB. . . . . . . . . . . . . . . . . . .

5.12 Reference and multiscale pressure solution after $1 \mathrm{~F}-\mathrm{MsRSB}$ cycle for single phase flow in the simple 3D model. Each fracture plane is logically partitioned into $12 \times 4$ blocks. . . . . . . . . . . . . . . .

5.13 Convergence property of the F-MsRSB + ILU (0) solver for various coarse grid resolutions per fracture plane. Each fracture plane is of size $100 \times 30$ at fine scale. . . . . . . . . . . . . . . .

5.14 Matrix grid with fracture planes and boundary conditions (a); Logarithm of permeability map (b) and matrix porosity $(\mathbf{c})$. . . . . . . .

5.15 Reference and multiscale pressure solution for single phase flow in the bed model with 2 degrees of freedom per fracture plane. . . . . . . . . . 28

5.16 5-spot well locations in the 3D Test Case 3, with 31 disconnected fracture networks. Shown on the right is the top view of the model. . . . .

5.17 Petrophysical properties for the $30 \times 110 \times 40$ domain sampled from the full SPE10 dataset . . . . . . . . . . . . . . . . . . .

5.18 Solution at the location of wells for the 3D Test Case 3. F-MsRSB solutions are shown for different tolerances of pressure solution, compared with reference solutions. 


\section{Chapter 1}

\section{Introduction}

Accurate simulation of multiphase flow in natural porous media represented on high-resolution computational grids is computationally demanding. Fine-scale petrophysical properties like permeability are often highly heterogeneous, change over several orders of magnitudes, and, in general, do not entail scale separation [4]. This computational challenge has motivated the development of several multiscale methods, which solve accurate coarse-scale systems constructed by the use of locally-computed basis functions [5-16]. Once the coarse-scale system is solved, its solution is interpolated into the original fine-scale resolution using the sub-resolution of the basis functions. Among the proposed multiscale methods, multiscale finite-volume (MSFV) methods not only provide mass-conservative solutions at fine-scale, which is a crucial property for convergent solution of transport equations, but also enable relatively simple inclusion of the type of multiphase flow equations seen in contemporary reservoir models $[6,17-22]$.

Multiscale methods compute approximate solution having the original fine-scale resolution so that their error (or residual) can be calculated with respect to the finescale discrete system. As such, one can achieve systematic strategies for reducing the error through iterative procedures that combine the multiscale solver with a finescale smoother [23-26]. Iterative multiscale methods are scalable and deliver massconservative solutions after any MSFV stage. The latter property makes them unique compared with alternative advanced solvers, such as multigrid methods [27]. Recent developments of the MSFV method include extensions to compressible and compositional non-linear displacements [28,29], unstructured grids [14,30], and fully-implicit simulations [31]. While these important developments, combined, cast a promising framework for next-generation simulators, they have been focused mainly on addressing challenges due to complex fluid physics, highly heterogeneous rock properties, and complex computational mesh geometries.

Many geological formations - including hydrocarbon reservoirs, underground water resources, and geothermal energy production fields - are naturally fractured. Fig. 1.1 shows outcrop examples of naturally fractured porous rocks. Outcrops give a fairly reasonable idea of the type of rocks one may find beneath the Earth's surface. It helps to understand how complex fracture networks, such as the ones shown in the figure, may influence fluid behaviour and pressure distribution throughout a reservoir made of the same type of deposits. Fractures are highly conductive channels which, for most 
practical purposes, exist in a lower-dimensional space compared to the porous matrix. Physical properties inside fractures and their length scales can be very different from those of the surrounding rock, adding significantly to the computational challenges, specially once realistic length scales and complex fracture network maps are considered. As a result, a variety of modelling approaches and numerical methods for different types of fractured reservoirs have been proposed [32-43,43-47]. Among them, the embedded fracture modelling approach $[1,37-39,48,49]$ benefits from independent grids for fracture and matrix, a promising approach for naturally fractured reservoirs and also for cases with dynamic fracture creations and closure of, e.g., geothermal systems. Note that small-scale fractures (smaller than fine-scale grid cells) are homogenized within the matrix porous rock, forming effective matrix conductivities [37]. This approach, similar to other discrete-fracture-modelling (DFM) approaches, lead to detailed fine-scale discrete systems (for matrix and fracture unknowns) with high contrasts within the entries, which are clearly much more challenging to be solved efficiently than non-fractured heterogeneous cases. Therefore, it is highly important to develop efficient multiscale methods for fractured formations.

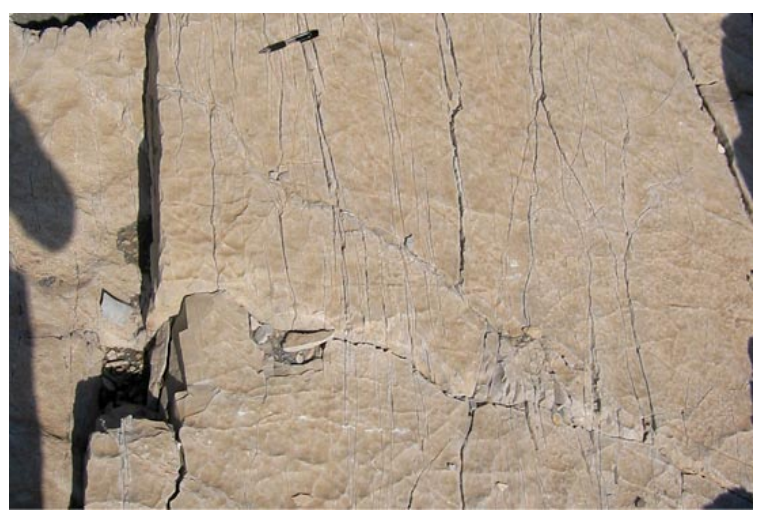

(a)

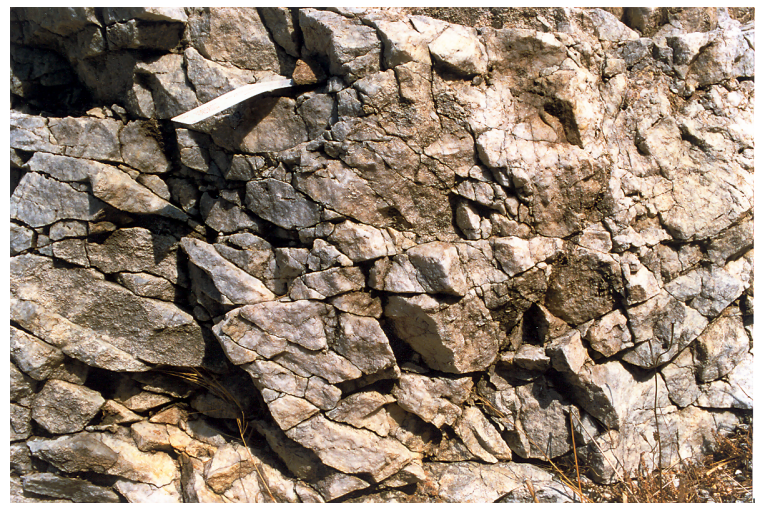

(c)

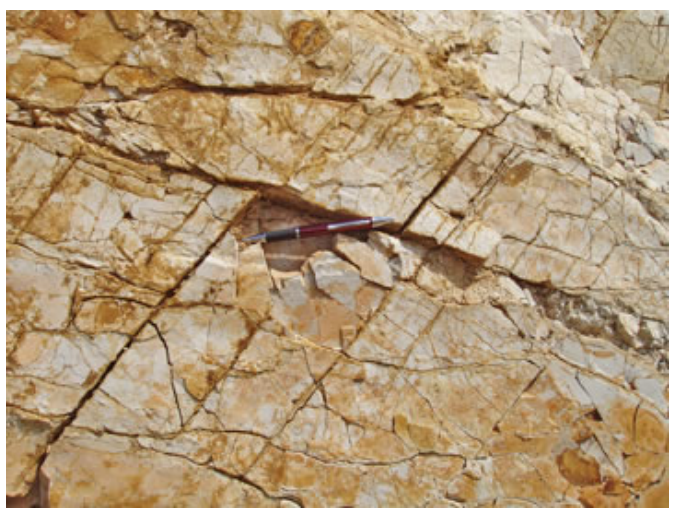

(b)

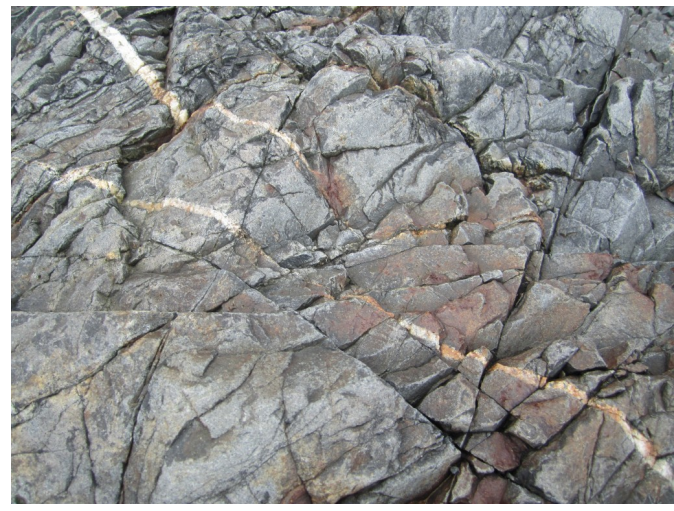

(d)

Fig. 1.1: Outcrop images of naturally fractured rocks

Early attempts at developing multiscale methods for fractured media were based on a mixed finite-element formulation in which high-conductive fractures were either represented explicitly as volumetric objects [40] or the fracture-matrix interaction was modelled by the Stokes-Brinkmann equations [41,50]. Within the MSFV framework, Hajibeygi et al. [1] developed the first multiscale method for fractured porous media, in 
which additional fracture basis functions were introduced to map each fracture network into one coarse-scale degree of freedom (DOF). Later, Sandve et al. [51] used the MSFV method to develop effective coarse-scale MINC-type model for fracture networks. Very recently, the treatment of fracture network was extended in a general formulation by proposing an algebraic multiscale solver for fractured media (F- $\underline{\text { AMS }}$ ) [2,52]. In the F-AMS, fracture basis functions were introduced on the basis of a coarsening ratio inside fracture domain, similar as in the matrix rock. Results of F-AMS, when only a few fracture DOFs were used, illustrated that such a multiscale map for fractured domains is quite efficient. Similar to all MSFV and AMS methods, F-AMS relies on coarse and dual-coarse grids imposed on the provided fine-scale grid cells. While the former is used to construct mass-conservative coarse-scale systems, the latter is employed to compute local basis functions. However, geological complexities and the use of complex grid geometries make the construction of these two coarse grids quite challenging. Recently, the multiscale restriction smoothed basis (MsRSB) method was devised by Møyner and Lie to overcome this complexity [3]. The MsRSB is unique in the way the basis functions are computed, yet leads to a stable and robust treatment of complex heterogeneous coefficients [53], as well as realistic flow physics for improved and enhanced oil recovery $[54,55]$. It is therefore favourable to use this method as a basis when seeking to extend multiscale simulation approaches for more complex fractured media.

In the work presented in this thesis, a multiscale restricted smoothed basis method for fractured media (F-MsRSB) is developed. Following F-AMS [2,52], F-MsRSB constructs basis functions for fractures and matrix in a general way, allowing for different level of coupling between them. In addition to F-AMS, though, F-MsRSB constructs its multiscale formulation on the basis of the MsRSB approach. This would facilitate its extension towards complex geometries while maintaining its efficiency for highly heterogeneous challenging scenarios such as the SPE10 comparative test case [56]. Unlike previous works, the performance of F-MsRSB is investigated for realistic fracture models with complex fracture networks. Transmissibility-weighted connectivity graphs of independent fractures are decomposed using the METIS software [57], leading to an automatic coarsening strategy for fractures. Following the traditional algebraic multiscale formulations, F-MsRSB can easily be adapted to account for complex physics such as compressibility [26] and gravity [58] as discussed in [54] for the MsRSB method. To facilitate implementation, specially for complex fracture networks, here, fracture cells are introduced into the discrete systems through non-neighbouring connections (NNC), see $[44,45,62]$.

Through several two- and three-dimensional cases with highly heterogeneous coefficients, F-MsRSB is found to efficiently compute approximate solutions of good quality. Furthermore, in order to allow for error control and reduction strategies, especially for multiphase flow scenarios, the method is combined with a fine-scale smoother, ILU(0) $[25,26,59]$. While low-frequency errors are resolved by the coarsescale system in F-MsRSB, the fine-scale smoother resolves high-frequency errors, the combination of which leads to an efficient (scalable) iterative multiscale solver for fractured media. These iterations are applied adaptively and infrequently just to maintain user-prescribed accuracy. Note that application of block smoothers near wells and fractures is also possible. It is easy to perform block smoothing using the solver strategy 
proposed and implemented for the numerical results of this thesis. Several multiphase flow cases are considered in which the adaptive iterative F-MsRSB is employed to efficiently compute high-quality solutions for the flow equations. All of these systematic single- and multiphase flow cases reveal that F-MsRSB is an efficient and versatile multiscale method for naturally fractured reservoirs with highly heterogeneous coefficients.

This thesis is structured as follows - The fine-scale discrete system for flow in fractured porous media is described in Chapter 2. Then, in Chapter 3, AMS and MsRSB are revisited. The development of F-MsRSB is presented in Chapter 4. Numerical results for single- and multi-phase flow for both 2D and 3D heterogeneous reservoirs are presented in Chapter 5. Finally, the thesis is concluded in Chapter 6. 


\section{Chapter 2}

\section{Governing equations and fine-scale system}

Mass conservation for a compressible phase $\alpha$ out of $n_{p h}$ phases, flowing in a porous medium in the absence of capillary forces reads,

$$
\frac{\partial\left(\phi \rho_{\alpha} S_{\alpha}\right)}{\partial t}-\nabla \cdot\left(\rho_{\alpha} \boldsymbol{\lambda}_{\alpha} \cdot(\nabla p-g \cdot \nabla z)\right)=q_{\alpha} \rho_{\alpha} \quad \forall \alpha \in\left[1, \ldots, n_{p h}\right],
$$

For incompressible fluids, density is constant with respect to space and time, leading to

$$
\frac{\partial}{\partial t}\left(\phi S_{\alpha}\right)-\nabla \cdot\left(\boldsymbol{\lambda}_{\alpha} \cdot \nabla p\right)=q_{\alpha} \quad \forall \alpha \in\left\{1, \ldots, n_{p h}\right\},
$$

where Darcy's law is employed to replace phase velocity $u_{\alpha}$ with pressure gradient $\nabla p$. Here, gravitational and capillary effects are both neglected. Moreover, $n_{p h}$ is the number of phases (and components), $S_{\alpha}$ and $\boldsymbol{\lambda}_{\alpha}$ are phase saturation and mobility, respectively. Note that $\boldsymbol{\lambda}_{\alpha}=\mathbf{k} k_{r \alpha} / \mu_{\alpha}$ holds, where the positive-definite permeability tensor, $\mathbf{k}$, is typically highly heterogeneous at multiple scales. Also, relative permeability, $k_{r \alpha}$ and phase viscosity, $\mu_{\alpha}$, are given functions of primary unknowns $p$ and $S$. These balance equations, along with the constraint that all phases fill the pore volume, i.e.,

$$
\sum_{\alpha=1}^{n_{p h}} S_{\alpha}=1,
$$

form a well-posed system of equations for $\left(n_{p h}+1\right)$ unknowns. Sequential approaches derive a pressure equation, which is solved first, then phase velocities are obtained to subsequently solve $n_{p h}-1$ transport equations (2.2). The $n_{p h}$-th saturation is obtained using the constraint (2.3). To obtain the pressure equation, i.e.,

$$
-\nabla \cdot\left(\boldsymbol{\lambda}_{t} \cdot \nabla p\right)=q_{t}
$$

phase balance equations (2.2) are summed up and the time-dependent term (accumulation) cancels out due to the constraint (2.3). Total mobility, $\boldsymbol{\lambda}_{t}$ and total source terms $q_{t}$ are obtained by summing their phase-wise counterparts.

For fractured porous media, following the hierarchical fracture model approach, small-scale fractures are homogenized and represented by an effective matrix permeability $\mathbf{k}^{m} \in \mathbb{R}^{n}$. Fractures with larger length scales are then explicitly represented 
with an embedded fracture modelling approach (EFM). Important to note is that the fracture elements can cross over matrix cells, or be confined at their interfaces. In the latter case, EFM reduces to alternative discrete fracture modelling approaches. Note that fractures are lower dimensional manifolds, due to their extremely small apertures, i.e., $\mathbf{k}^{f} \in \mathbb{R}^{n-1}$. In this case, pressure equation can be expressed as

$$
-\nabla \cdot\left(\boldsymbol{\lambda}_{\mathbf{t}} \cdot \nabla p\right)^{m}+\psi^{m f}=q_{t}^{m} \quad \text { on } \quad \Omega^{m} \subset \mathbb{R}^{n}
$$

and

$$
-\nabla \cdot\left(\boldsymbol{\lambda}_{\mathbf{t}} \cdot \nabla p\right)^{f}+\psi^{f m}=q_{t}^{m} \quad \text { on } \quad \Omega^{f} \subset \mathbb{R}^{n-1}
$$

where superscripts ${ }^{m}$ and ${ }^{f}$ represent matrix and fracture quantities, respectively. Mass exchange between fracture and matrix cells, $\psi^{m f}$ and $\psi^{f m}$, are modelled as

$$
\psi^{f m}=C I \boldsymbol{\lambda}_{t}^{*}\left(p^{f}-p^{m}\right)=-\psi^{m f},
$$

where $C I$ is the fracture-matrix conductivity index [1,39]. Interaction of a matrix element $i$ and a fracture element $j$ is defined as

$$
C I=\frac{A_{i-j}}{\langle d\rangle_{i-j}}
$$

where $A_{i-j}$ is the fracture plate area and $\langle d\rangle_{i-j}$ is the average distance between $i$ and $j$. More information about EFM and the calculation of its parameters can be found in [1]. An advantage of EFM is that the fracture and matrix grids are independent and, thus, suited for many realistic scenarios such as naturally fractured reservoirs and dynamic fracture generation and closures.

Finite volume discretization of Eq. (2.5) and Eq. (2.6) leads to the fine-scale system $\mathbf{A p}=\mathbf{q}$ for matrix and fracture pressure unknowns, i.e.,

$$
\left[\begin{array}{ccc}
A_{m m} & A_{m f} & A_{m w} \\
A_{f m} & A_{f f} & A_{f w} \\
A_{w m} & A_{w f} & A_{w w}
\end{array}\right]\left[\begin{array}{l}
p^{m} \\
p^{f} \\
p^{w}
\end{array}\right]=\left[\begin{array}{l}
q^{m} \\
q^{f} \\
q^{w}
\end{array}\right]
$$

where ${ }^{w}$ super-index denotes external well (source) terms [4]. Obviously, $A_{f w}$ and $A_{w f}$ will be zero, if no well is drilled into the fracture domain.

The formulation of this thesis is developed into the open-source Matlab simulator MRST [60,61], in which fractures are introduced using non-neighbouring connections (NNC) $[44,45,62]$. In addition, a sequential implicit strategy has been followed for the multiphase flow studies depicted in this thesis.

Large-scale heterogeneous formations with complex fracture network maps, along with high contrasts between fracture and matrix properties, make Eq. (2.9) quite challenging to solve using any classical numerical methods. To resolve this computational challenge, a multiscale restriction smoothed basis method for fractured media (F-MsRSB) is developed. The F-MsRSB benefits from the previously developed multiscale methods for fractured media $[1,2]$ and the MsRSB formulation for non-fractured systems [3]. 


\section{Chapter 3}

\section{Multiscale Restriction Smoothed Basis Method (MsRSB)}

In this chapter, MsRSB [3] is described in algebraic form [25, 26]. More precisely, a general algebraic formulation of multiscale methods is first introduced before we continue to describe the specific prolongation and restriction operators of the MsRSB method. This chapter will cast the foundation of the next chapter in which the novel development of this thesis, i.e., F-MsRSB, is presented.

\subsection{Algebraic multiscale formulation}

To avoid solving $A p=q($ Eq. (2.9)) directly on the fine scale, multiscale methods introduce a coarse-scale system,

$$
\underbrace{(\mathcal{R} A \mathcal{P})}_{A^{c}} p^{c}=\underbrace{\mathcal{R} q}_{q^{c}}
$$

which has much smaller size (i.e., $n_{c} \times n_{c}$ ) than the original fine-scale system (i.e., $\left.n_{f} \times n_{f}\right)$. The Restriction operator $\mathcal{R}$ maps the fine-scale system into the coarse scale, while the Prolongation operator $\mathcal{P}$ interpolates the coarse-scale solution into the finescale original resolution [18]. Once the coarse-scale system is solved, an approximate fine-scale solution $p^{\prime}$ is obtained by an interpolating formulation, i.e.,

$$
p^{\prime}=\mathcal{P} p^{c}
$$

Combining Eq. (3.1) and Eq. (3.2), the AMS procedure can be summarized as

$$
p \approx p^{\prime}=\underbrace{\mathcal{P}(\mathcal{R} A \mathcal{P})^{-1} \mathcal{R}}_{M_{m s}^{-1}} q .
$$

Similar to AMS, MsRSB imposes a coarse grid on top of the provided fine-scale grid. Inside each coarse grid cell (coarse control volumes), a fine-scale grid cell is selected as coarse node. Coarse cells $\Omega_{K}^{c}$ form a non-overlapping partition of the domain.

For the restriction operator, there are two different choices, either to use $\mathcal{R}=\mathcal{P}^{T}$, which will lead to a Galerkin-type formulation, or to use a finite-volume restriction 
procedure [6], which can be stated as discrete integration operator over coarse control volumes $\Omega^{c}$, i.e.,

$$
\mathcal{R}_{i, K}= \begin{cases}1 & \text { if } \Omega_{i} \subset \Omega_{K}^{c} \\ 0 & \text { otherwise. }\end{cases}
$$

As shown in $[3,53]$, the MsRSB method is not very sensitive to the choice of restriction and herein we use the finite-volume operator to ensure that we can reconstruct fine-scale conservative velocities.

The prolongation operator is constructed by solving localized flow problems, and the way these flow problems are set up varies from one method to another. However, in all multiscale methods, the prolongation operator $\mathcal{P}$ is defined so that it stores basis function $\Phi_{K}$ associated with coarse block $\Omega_{K}^{c}$ in its $K$-th column, i.e.,

$$
\mathcal{P}_{i, K}=\Phi_{K}\left(\mathbf{x}_{i}\right) \quad \forall i \in\left\{1, \ldots, n_{f}\right\}, \forall K \in\left\{1, \ldots, n_{c}\right\}
$$

Here, $\Phi_{K}\left(\mathbf{x}_{i}\right)$ is the value of basis function $\Phi_{K}$ at $i$-th fine-grid cell, $\mathbf{x}_{i}$.

Both the original MSFV method [6] and its state-of-the-art extension (AMS) $[25,26]$ rely on a secondary coarse partition, defined as the dual to the primal coarse grid, over which the basis functions $\Phi_{K}$ are locally computed. While it is possible to extend conservative multiscale methods based on a dual-grid formulation to stratigraphic and other types of unstructured grids $[21,30,51,63]$, it has proved to be difficult, if possible, to develop satisfactory dual-primal partitions for a grid with complex geometries. Moreover, localization errors induced by strong permeability contrasts across block boundaries introduce instabilities in the corresponding multipoint coarse-scale stencil. This motivated the development of a multiscale two-point flux-approximation formulation [14], in which an implicitly defined dual grid is used to compose elementary flow solutions into localized basis functions. In the MsRSB method, however, local supports for basis functions are defined based on interaction regions, which are relatively simple to define even for very complex grids. Once these interaction regions are obtained, restriction-smoothed basis functions are computed by employing a modified form of the damped-Jacobi smoothing approach, similar as in smoothed-aggregationbased multigrid methods [64-66]. In the following sections, the MsRSB interaction regions and basis functions are briefly explained. Detailed explanations can be found in $[3,53]$.

\subsection{Coarse grid and interaction regions}

Basis function $\Phi_{K}$ can have non-zero values only in the interaction region $I_{K}$. For the specific case of MSFV, e.g., $I_{K}$ reduces to the set of dual-coarse grid cells overlapping with the $K$-th coarse block. Thus, by construction, the basis function $\Phi_{K}$ and consequently the $K$-th column of $\mathcal{P}$ is set to 0 outside $I_{K}$.

Interaction region of a coarse block $\Omega_{K}^{c}$ (see Fig. 3.1) is constructed by creating a local triangulation, using cell and shared-face centroids of all immediate geometrical neighbours of $\Omega_{K}^{c}$ [3]. This ensures that for a Cartesian grid without fractures, the coarse system has the same multipoint flux stencil as in the original MSFV method. 
Interaction regions for fractured coarse blocks are, because of their (potentially severe) geometrical complexities, computed by a different procedure, which will be described in the next chapter (See Table 4.1).

Interaction boundary $B_{K}$ is defined as the set of all fine cells that are topological neighbours of the outermost cells in the interaction region $I_{K}$. This leads to the definition of a global boundary $G$ which is a union of all $B_{K} \forall K \in\left\{1, \ldots, n_{c}\right\}$, i.e.,

$$
G=B_{1} \cup B_{2} \cup \cdots \cup B_{n_{c}} .
$$

Fig. 3.1 illustrates $B$ and $G$ for a $2 D$ rectangular cartesian and an unstructured hexagonal grid. For a Cartesian grid geometry, $G$ becomes equivalent to the set of all dual-coarse boundary cells, i.e., similar to the classical MSFV method. Finally, indices of all coarse blocks whose interaction regions overlap with each fine cell $i$ inside $G$, i.e. $\mathbf{x}_{i}$, are stored in the set $H_{i}$, i.e.,

$$
H_{i}=\left\{K \mid \mathbf{x}_{i} \in I_{K}, \mathbf{x}_{i} \in G\right\} .
$$

\subsection{MsRSB prolongation operator}

As mentioned earlier, basis functions are calculated iteratively, having non-zero values only inside the corresponding interaction regions. To obtain basis functions, a constant value of 1 for each coarse block is set as initial value, i.e.,

$$
\mathcal{P}_{i, K}^{0}= \begin{cases}1 & \text { if } \mathbf{x}_{i} \in \Omega_{K} \\ 0 & \text { otherwise }\end{cases}
$$

This is followed by computing the iterative increments

$$
\hat{d}_{K}=-\omega D^{-1} A \mathcal{P}_{K}^{n},
$$

where $A$ is the fine scale system, $D$ is the diagonal entries of $A(D=\operatorname{diag}(A))$, and $\omega$ is a relaxation (or damping) parameter, which is set to $2 / 3$ for all simulations of this thesis.

Due to the local supports for basis functions, the increments $\hat{d}_{K}$ must be restricted to have non-zero values only inside $I_{K}$. This is done by setting $\mathcal{P}_{K}^{n}$ outside the interaction region to 0 and normalizing all other basis functions that have non-zero values at the boundary cells $B_{K}$, i.e.,

$$
d_{i K}= \begin{cases}\frac{\hat{d}_{i K}-\mathcal{P}_{i K} \sum_{J \in H_{i}} \hat{d}_{i J}}{1+\sum_{J \in H_{i}} \hat{d}_{i J}} & \text { if } i \in I_{K}, i \in G \\ \hat{d}_{i K} & \text { if } i \in I_{K}, i \notin G \\ 0 & \text { if } i \notin I_{K} .\end{cases}
$$

This modified increment is now used to update the prolongation operator, i.e.,

$$
\mathcal{P}_{K}^{n+1}=\mathcal{P}_{K}^{n}+d_{K}
$$




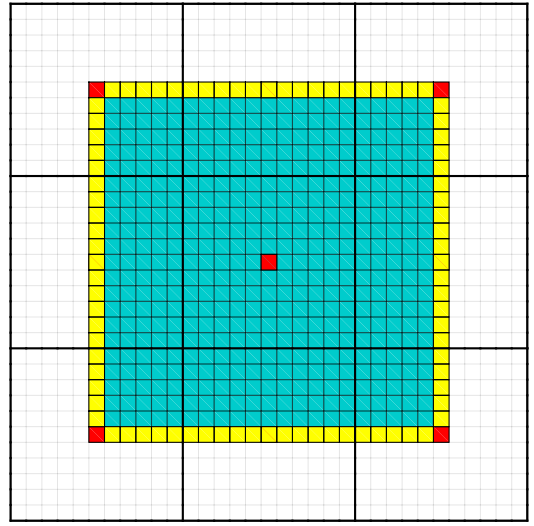

(a) Interaction boundary $B_{(2,2)}$

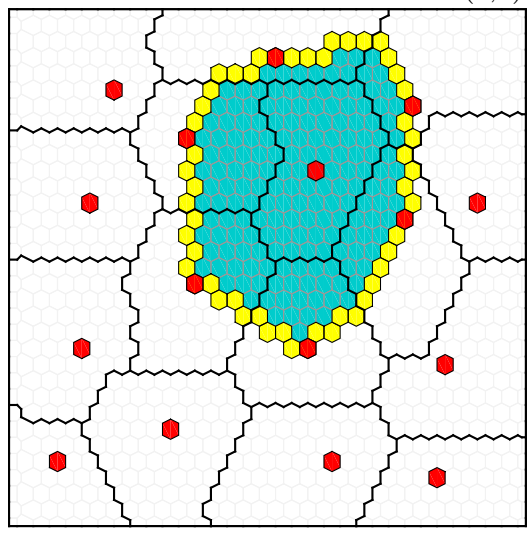

(c) Interaction boundary $B_{(3,3)}$

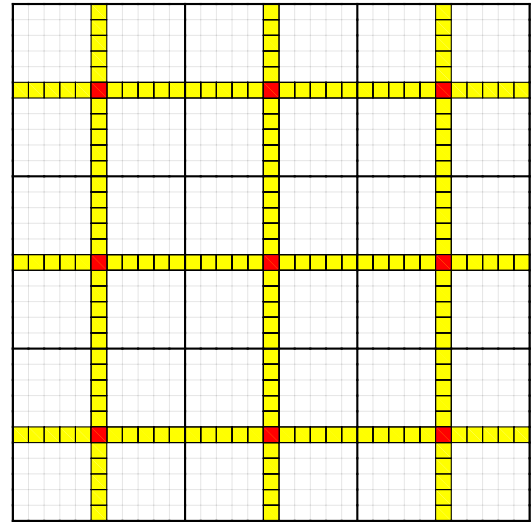

(b) Global boundary $G$

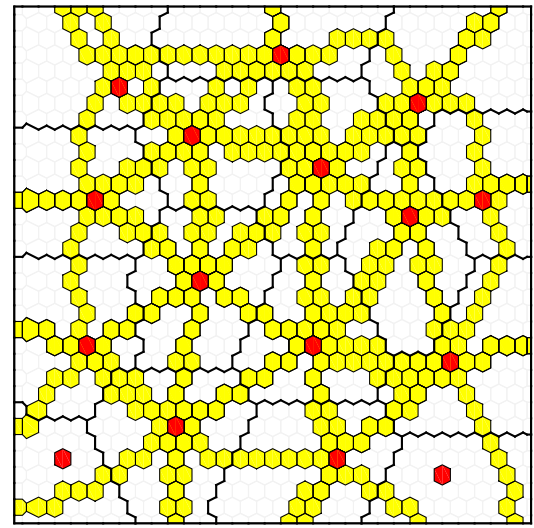

(d) Global boundary $G$

Fig. 3.1: Highlighted cells in turquoise colour are the interaction region for a coarse node (a and $\mathbf{c}$ ). Also shown in yellow, in the same plots (a and $\mathbf{c}$ ), are the interaction boundary cells. Shown on the right are the global boundary cells $G$, highlighted in yellow (b and $\mathbf{d}$ ). The top row shows a rectangular grid with a uniform coarse partition, while the bottom row presents a hexagonal grid with an unstructured coarse partition.

To measure convergence of the basis functions, a local error $e_{K}$ is defined outside $G$, and basis functions are assumed to be converged if $\|e\|_{\infty} \leq$ tol, where

$$
e_{K}=\max _{i}\left(\left|\hat{d}_{i K}\right|\right), \quad i \notin G \text {. }
$$

Note that modification proposed in Eq. (3.10) enforces a local support for the final basis functions to be used to construct a sparse prolongation operator. Fig. 3.2, Fig. 3.3 and Fig. 3.4 show the effect the modification in Eq. (3.10) has on basis function calculation for homogeneous $1 \mathrm{D}$, homogeneous $2 \mathrm{D}$, and several $2 \mathrm{D}$ permeability fields, respectively. MSFV basis functions are also shown in comparison with restriction smoothed basis functions in the latter.

Without the modification proposed in Eq. (3.10), each basis function would iteratively grow to spread across the entire domain. For a conservative discretization scheme such as finite volume, row sum of system matrix $\mathbf{A}$ will always be zero, i.e. $\sum_{j} \mathbf{A}_{i j}=0$. By virtue of using disjoint or non-overlapping coarse partitions, Eq. (3.8) ensures that the row sum of the prolongation matrix is always 1 . It can then be mathematically verified that every successive Jacobi iteration over this initial guess would 
always honour a partition of unity in the prolongation matrix, i.e. $\sum_{K} \mathcal{P}_{i K}=1$. For a homogeneous domain, this would entail $\mathcal{P}_{i K}=1 / n_{c} \forall i \in\left\{1, \ldots, n_{f}\right\}, K \in\left\{1, \ldots, n_{c}\right\}$ since every fine cell receives an equal contribution from each of the $n_{c}$ global basis functions.

Restricting the spread of basis functions in this manner is similar to the concept of a reduced boundary condition at dual cell boundaries as in classical MSFV. It is important to note that this modification localizes each basis function and is ultimately the source of error in the multiscale solution.
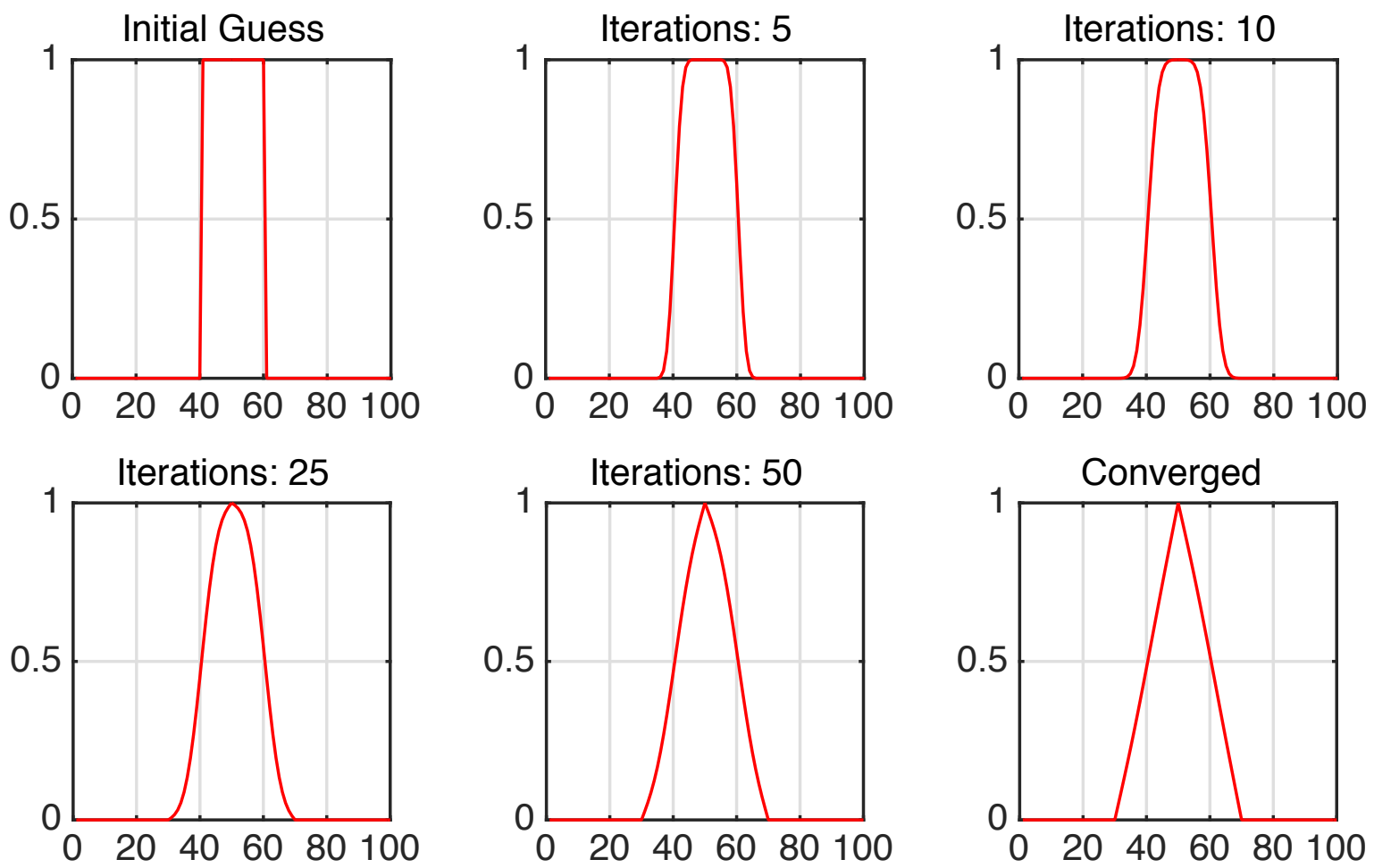

Fig. 3.2: Restriction smoothed basis function computed iteratively over a $100 \mathrm{~m}$ long 1D homogeneous domain with $20 \mathrm{~m}$ coarse blocks. Vertical axis gives the basis function value for the $3^{\text {rd }}$ coarse block. Tolerance for convergence $=10^{-3}$. 

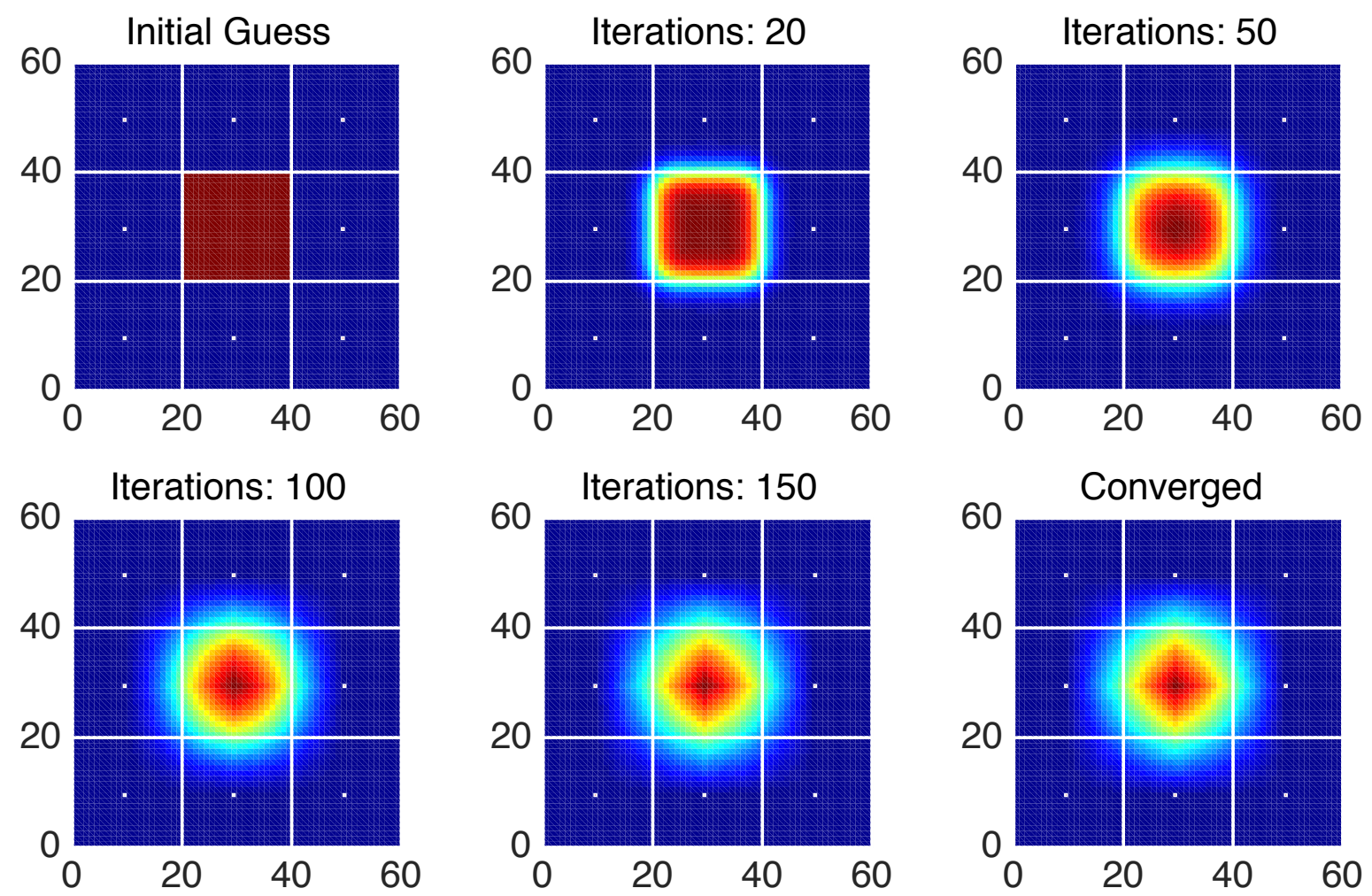

Fig. 3.3: Restriction smoothed basis function for coarse block $(2,2)$ computed iteratively over a $60 \times 60$ homogeneous domain with $3 \times 3$ coarse blocks. Tolerance for convergence $=10^{-3}$. 


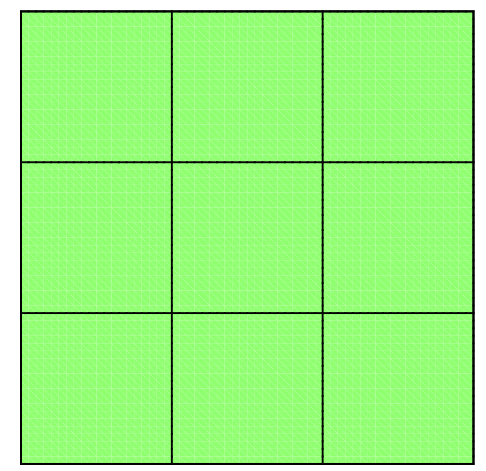

(a) Homogeneous

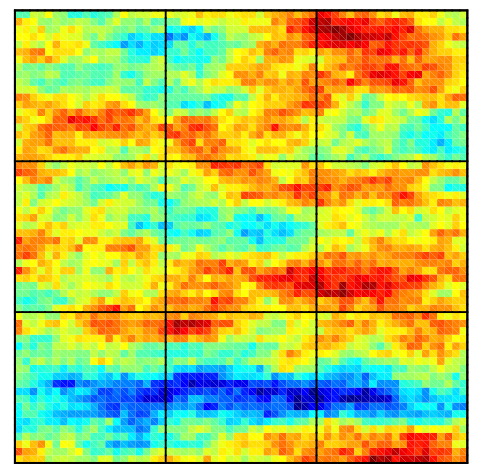

(b) Patchy

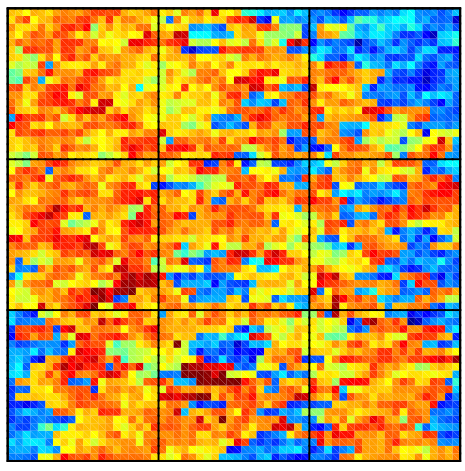

(c) Channelized

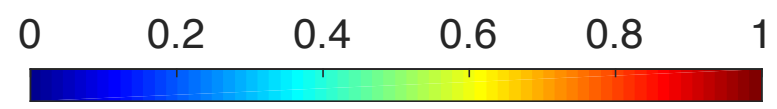

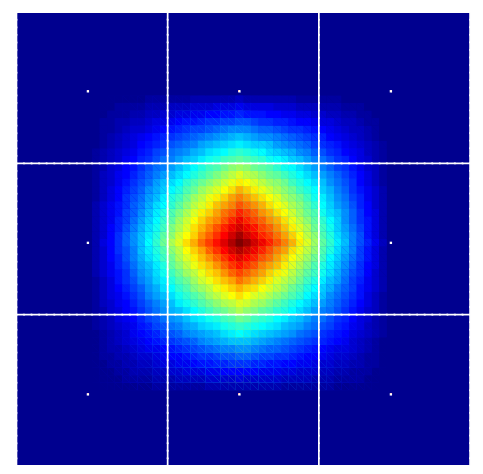

(d)

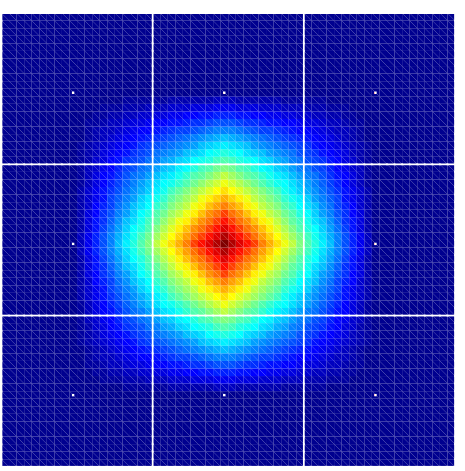

(g)

MsRSB basis functions

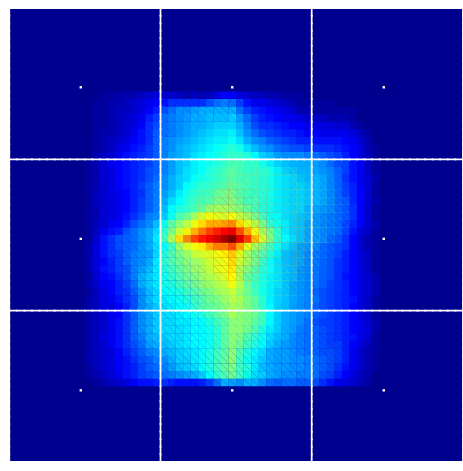

(e)

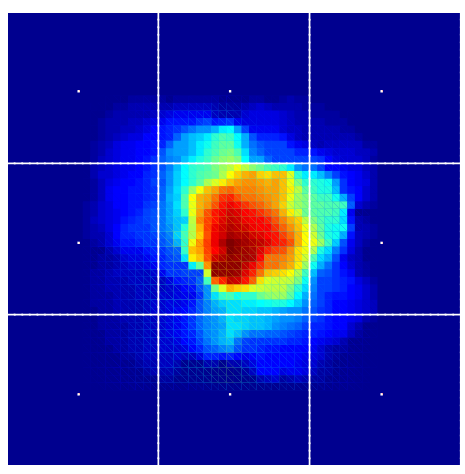

(f)

MSFV basis functions

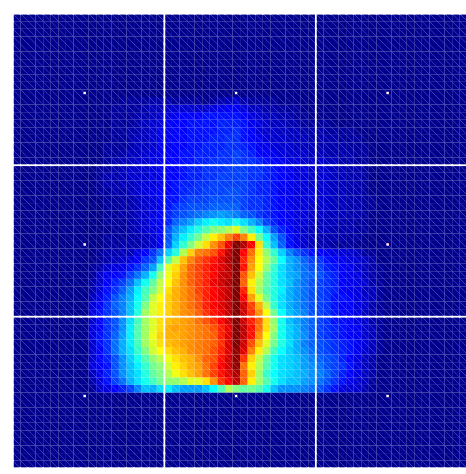

(h)

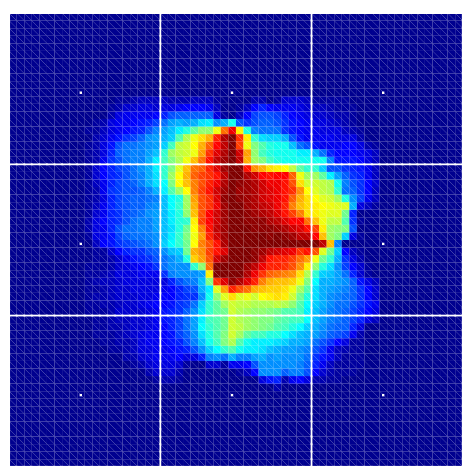

(i)

Fig. 3.4: Illustration of the restriction smoothed basis functions (d-f) and classical MSFV basis functions (g-i) for three different permeability fields: homogeneous (a), heterogeneous patchy-field (b) and heterogeneous channelized field (c). 


\section{Chapter 4}

\section{MsRSB for Fractured Media (F-MsRSB)}

In densely fractured reservoirs, phase transport predominantly takes place through fractures. Their permeability is generally several orders of magnitude higher than the matrix average and as a result, they alter the flux field across a reservoir quite significantly. Therefore, it would be preferable to have a multiscale formulation for fractures in addition to one in the matrix. Note that by virtue of fracture connectivity defined through NNC, the MsRSB solver for non-fractured media can handle presence of fractures without any special treatment. However, this is neither preferable nor practical because without a separate coarsening inside fractures, the conventional multiscale formulation will not be able to accurately account for their impact on reservoir pressure.

\subsection{Algebraic formulation}

The F-MsRSB method is devised on the idea of introducing basis functions for both matrix and fracture domains, similar to F-AMS [2], i.e., $p \approx p^{\prime}=\left[\begin{array}{ll}p_{m}^{\prime} & p_{f}^{\prime}\end{array}\right]^{T}$, where

$$
p_{m}^{\prime}=\sum_{j=1}^{n_{c m}} \Phi_{j}^{m, m} p_{j}^{c, m}+\sum_{i=1}^{N_{f n}} \sum_{j=1}^{n_{c f_{i}}} \Phi_{j}^{f_{i}, m} p_{j}^{c, f_{i}}
$$

and

$$
p_{f}^{\prime}=\sum_{j=1}^{n_{c m}} \Phi_{j}^{m, f_{i}} p_{j}^{c, m}+\sum_{i=1}^{N_{f n}} \sum_{j=1}^{n_{c f_{i}}} \Phi_{j}^{f, f_{i}} p_{j}^{c, f_{i}},
$$

where $p_{m}^{\prime}$ and $p_{f}^{\prime}$ are F-MsRSB approximate matrix and fracture pressure at fine-scale, respectively. In addition coarse-scale solutions, in matrix and fracture, are denoted as $p^{c, m}$ and $p^{c, f}$, respectively. There exist $n_{c m}$ matrix coarse blocks, and the fracture network $i$ has $n_{c f_{i}}$ coarse cells. Each fracture network can consist of several connected fracture plates (lines for 2D domains), and in total, there are $N_{f n}$ number of disconnected fracture networks. Moreover, $\Phi^{m, m}$ and $\Phi^{m, f}$ are basis functions for matrix coarse cells with superscripts ${ }^{m, m}$ and ${ }^{m, f}$ denoting values in the matrix and fracture domains, respectively. Both $\Phi^{f, f}$ and $\Phi^{f, m}$ are fracture basis functions with superscript $f, f$ representing the values inside the corresponding fracture network and superscript $f, m$ denoting contributions inside the matrix domain. Hence, the prolongation operator 
can be written as

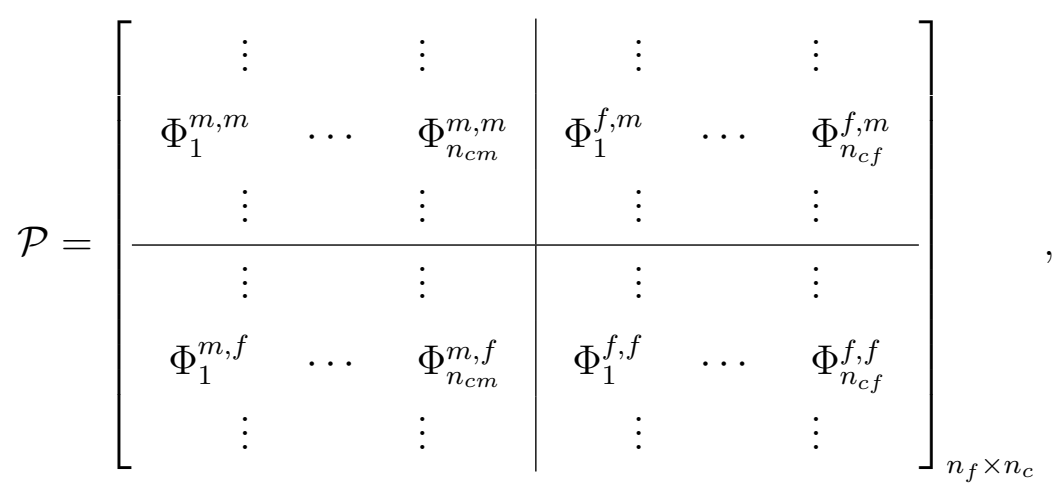

where $n_{f}=\left(n_{f}^{m}+n_{f}^{f}\right)$ and $n_{c}=\left(n_{c m}+n_{c f}\right)$ are total degrees of freedom (matrix and fractures) at fine and coarse scales.

Generally, fractures are much more conductive than the matrix rock. Full consideration of both fracture and matrix coarse solutions, $p_{m}^{c}$ and $p_{f}^{c}$, for interpolated fracture pressure, $p_{f}^{\prime}$, can lead to improved convergence properties. However, such an approach results in much denser prolongation operators. Therefore, the improvement in convergence rate may not necessarily offset the computational cost of additional operations associated with it. Numerical studies of F-AMS for 3D problems (considering CPU time), support the idea of eliminating the effect of matrix coarse pressure in the fracture pressure interpolation, i.e., setting $\Phi^{m, f}=0$. In this thesis, the same sparse operator is considered.

Next, the interaction region and the procedure for calculating basis functions for fractured media are explained.

\subsection{Interaction regions and basis functions}

The interaction region for each fracture coarse block is generated based on a topological distance or connectivity based algorithm. More precisely, the interaction region for a fracture coarse cell includes all fine cells located inside the sphere (circle in 2D) with radius $d=\lceil\beta / a\rceil$ residing in a radial index space. The constant factor $\beta$ is in the range of $(0,1)$, and $a$ is the fracture aperture. It is clear that the interaction region will include no fine-cell except those overlapping with the fractures, if $\beta=0$ is considered. In the other extreme, i.e., if $\beta=1$, all fine cells within the distance equal to the inverse of aperture will be considered. For the numerical examples studied in this thesis, the value of $\beta=1 / 4$ is used. An overview of the procedure to generate the fracture interaction region is presented in Table 4.1 .

Fig. 4.1 illustrates coarse grids and interaction regions inside which basis functions are compactly supported for a test case with $30 \times 30$ matrix and 20 fracture cells. Furthermore, Fig. 4.2 shows basis functions inside the matrix rock (belonging to both fracture and matrix coarse nodes). 
Table 4.1: Algorithm for generating fracture interaction regions

Initialize: $A=$ Adjacency matrix for the fine-scale system, $d=\lceil\beta / a\rceil$ and $m=1$

1: for $J \in\left\{1, \ldots, n_{c f}\right\}$ do

2: $\quad I_{i, J}=1$ if $\mathbf{x}_{i} \in \Omega_{J}^{c}, \quad I_{i, J}=0$ otherwise

3: $\quad$ while $m<d$ do

4: $\quad I_{J}=A \times I_{J}$

5: $\quad m=m+1$

6: $\quad$ end while

7: end for

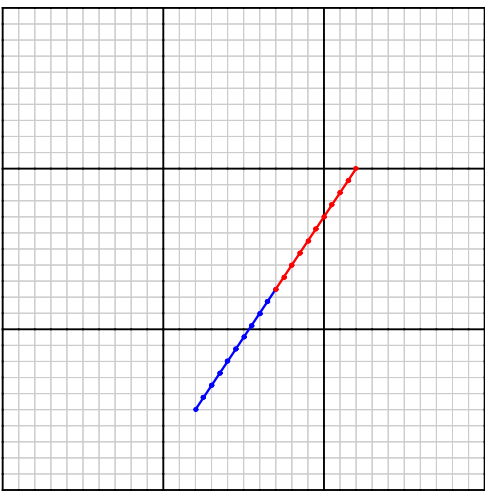

(a)

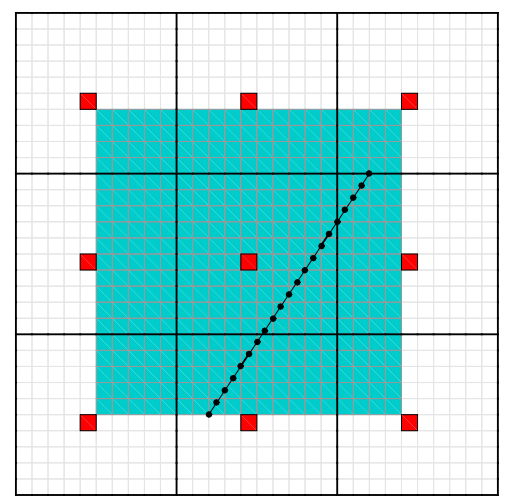

(b)

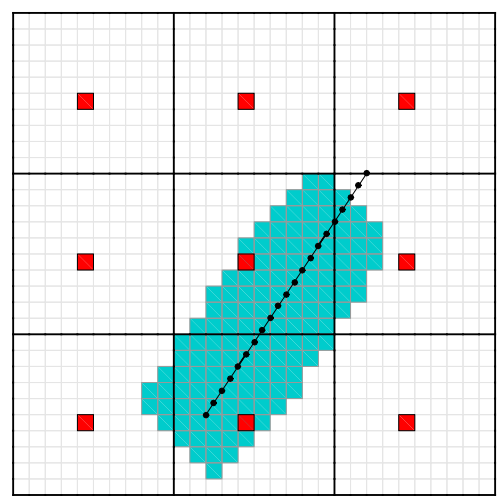

(c)

Fig. 4.1: Illustration of multiscale grids for matrix and fracture (a) with interaction regions for matrix (b) and fracture (c) for a case with $30 \times 30$ matrix and 20 fracture fine-scale cells. Multiscale coarse grid contains $3 \times 3$ matrix blocks and 2 blocks inside the fracture. Here, $a=1 / 25$ and, consequently, $d=7$. 


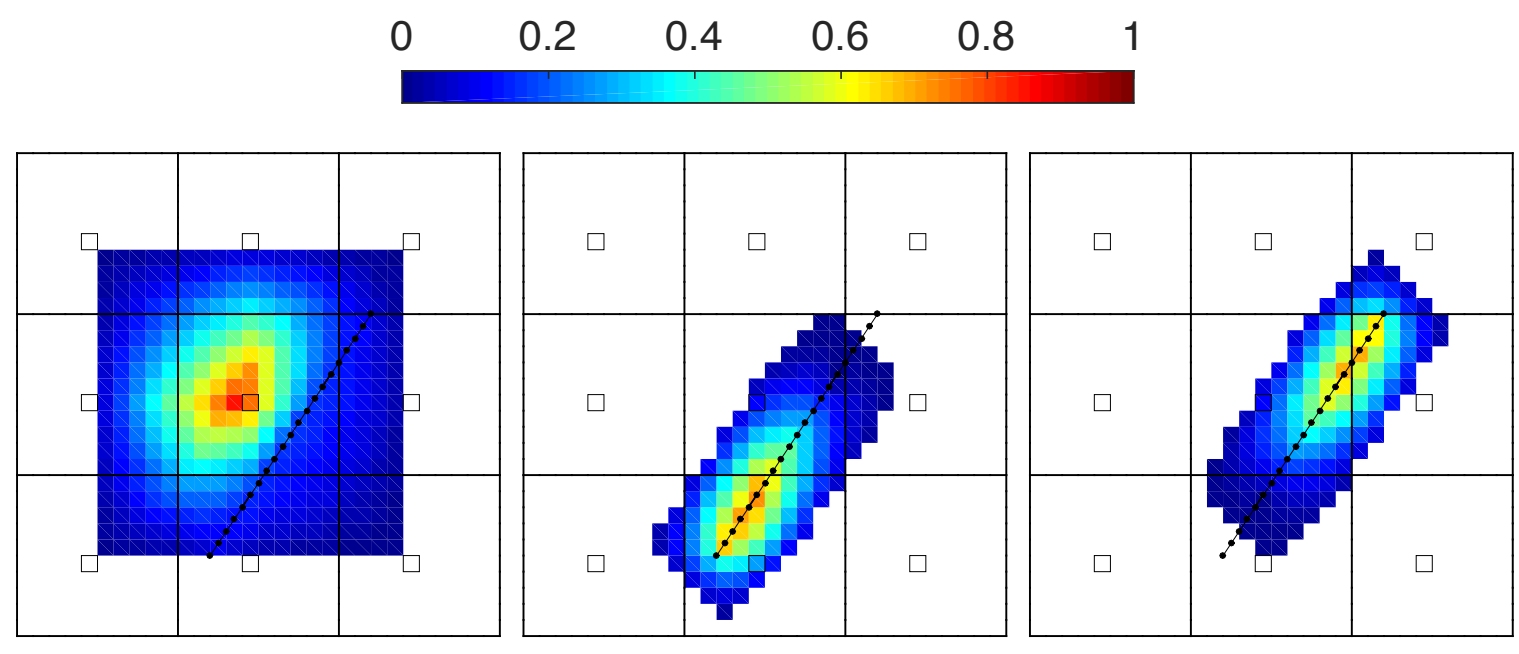

(a) $\Phi_{5}^{m, m}$

(b) $\Phi_{1}^{f, m}$

(c) $\Phi_{2}^{f, m}$

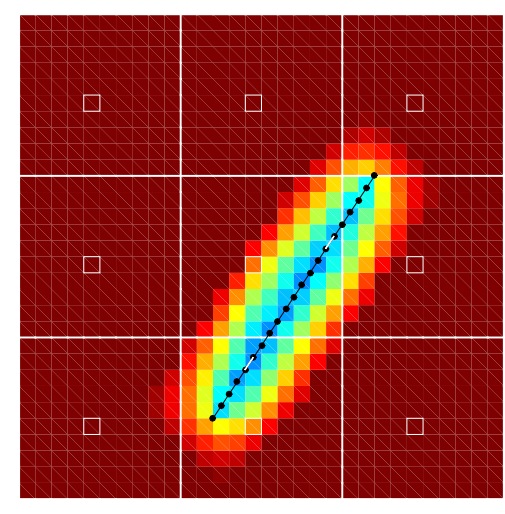

(d) $\sum_{j=1}^{n_{c m}} \Phi_{j}^{m, m}$

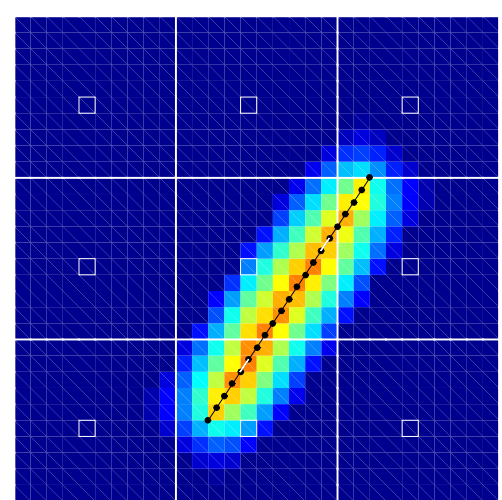

(e) $\sum_{j=1}^{n_{c f}} \Phi_{j}^{f, m}$

Fig. 4.2: Matrix and fracture basis functions for the system described in Fig. 4.1 


\section{Chapter 5}

\section{Numerical Results}

The developed F-MsRSB method is implemented and integrated with the free, open-source Matlab Reservoir Simulation Toolbox (MRST) [60,61,67,68]. In this chapter, we investigate the performance of F-MsRSB for many challenging test cases. The numerical examples involve both 2D and 3D heterogeneous media. Next, the sensitivity of the method to coarse-grid resolution for fracture domain (coarse degrees of freedom - DOF) is studied. Then, its performance for heterogeneous rock formations is analysed through a realistic fracture map obtained from an outcrop, and for a statistically generated fracture map. Using an outcrop map is a unique test case in the literature of multiscale methods for fractured media. For the statistical map, we use METIS [57] to generate an unstructured partition for a fine-scale hexagonal grid representing the matrix rock. Finally, three test cases with 3D heterogeneous matrix properties along with 2D fracture plates are considered, in order to provide the scientific community with a reliable assessment of the devised F-MsRSB method.

\subsection{Sensitivity to Coarse DOF in fracture}

In this test case, single phase flow in a $100 \times 100 \mathrm{~m}^{2}$ homogeneous domain is considered. The effect of coarsening ratio in fracture domain on the multiscale solution accuracy and on its preconditioning properties is studied. The matrix permeability is set to 1 Darcy and $k^{f} / k^{m}=10000$. Fluid viscosity is $1 \mathrm{cP}$. The matrix contains $100 \times 100$ fine-scale grid cells. As shown in Fig. 5.1, the matrix contains one fracture network containing 200 fine-scale grid cells. A coarsening ratio of $10 \times 10$ is fixed for matrix domain, while the coarsening ratio for fracture is varied from 1 to 50 (some cases are shown in Fig. 5.2). By increasing the coarse DOF in the fracture, the FMsRSB pressure solution improves (Fig. 5.3). This finding is consistent with that of F-AMS [2].

Fig. 5.3 shows pressure solutions after 1 multiscale cycle for different fracture coarsening ratios. As a quantitative error measurement, F-MsRSB pressure error is calculated using a scaled $2^{\text {nd }}$ norm given as

$$
\epsilon_{p}=\sqrt{\frac{\sum_{i \in n_{f}}\left(p_{i}^{f s}-p_{i}^{m s}\right)^{2} \times \Omega_{i}}{\sum_{i \in n_{f}}\left(p_{i}^{f s}\right)^{2} \times \Omega_{i}}} .
$$




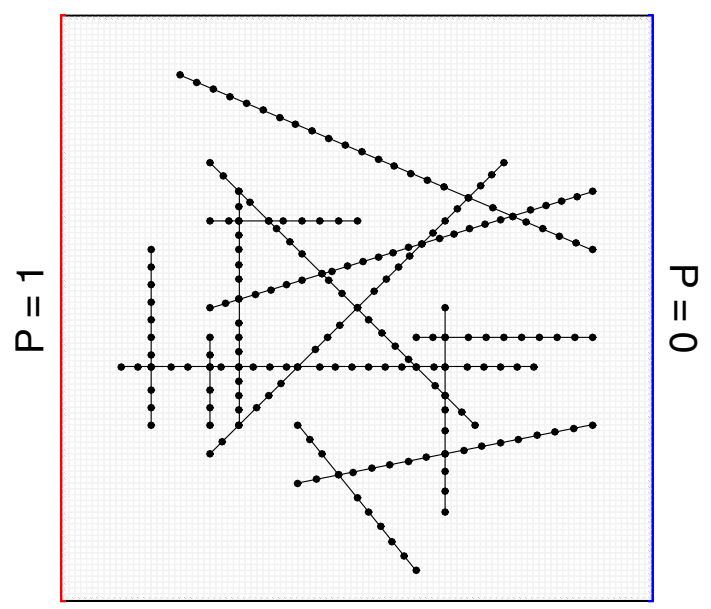

Fig. 5.1: First test case which contains $100 \times 100$ matrix and 200 fracture cells at fine scale, with homogeneous $2 \mathrm{D}$ rock formation. The left and right boundaries are subject to Dirichlet values of 1 and 0 , respectively.

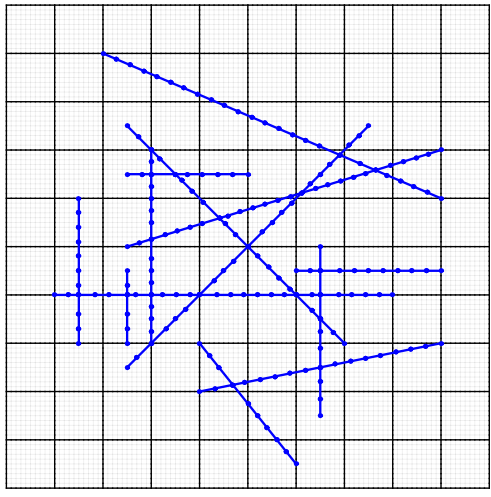

(a) 1 DOF in fracture

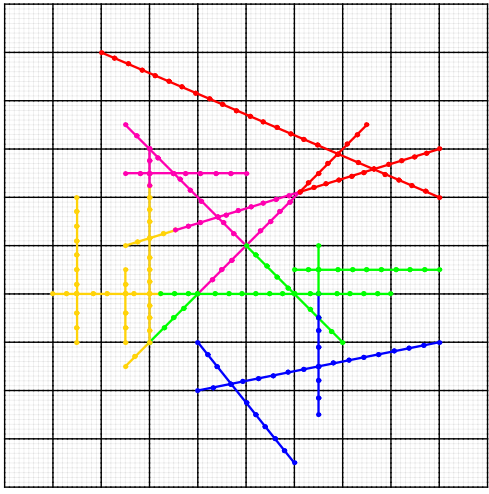

(b) 5 DOF in fracture

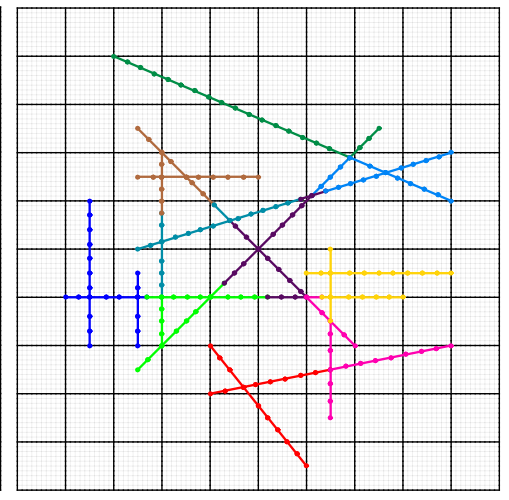

(c) 10 DOF in fracture

Fig. 5.2: F-MsRSB coarse grids for matrix and fractures. The matrix coarse grid consists of $10 \times 10$ fine cells, while the fracture coarse cells are varied from 1 (200 fine cells in 1 block) to 5 (40 cells in each block) and 10 (20 cells in each block).

For different fracture coarse-scale grid sizes, the F-MsRSB errors are also provided in Fig. 5.3. Similar to F-AMS, the condition number of the F-MsRSB coarse system also improves with increasing DOF in fracture at coarse scale. This leads to higher iterative convergence rates, as shown in Fig. 5.4, if F-MsRSB is combined with ILU(0) in an iterative multiscale procedure [2]. Convergence is determined on the basis of setting a threshold value for the scaled residual norm, i.e., $\left\|r_{b}\right\|_{2}=\|A p-q\|_{2} /\|q\|_{2}$.

\subsection{F-MsRSB for heterogeneous fractured media}

To study the F-MsRSB for heterogeneous fractured media, two fracture maps are considered: (1) the fracture map is extracted from an outcrop of dimensions $246.3 m \times 283.1 m$; (2) a statistical fracture model for an unstructured perpendicular bisector or PEBI grid is generated. The heterogeneous rock property is assumed to include homogenized small-scale fractures in addition to the conventional heterogeneity variations in the matrix rock. 


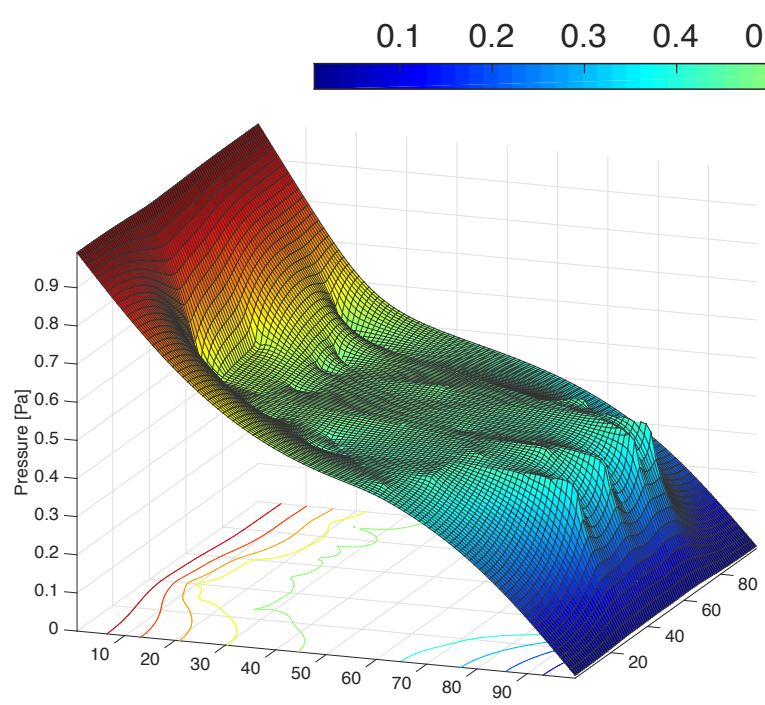

(a) Fine-scale

Reference solution

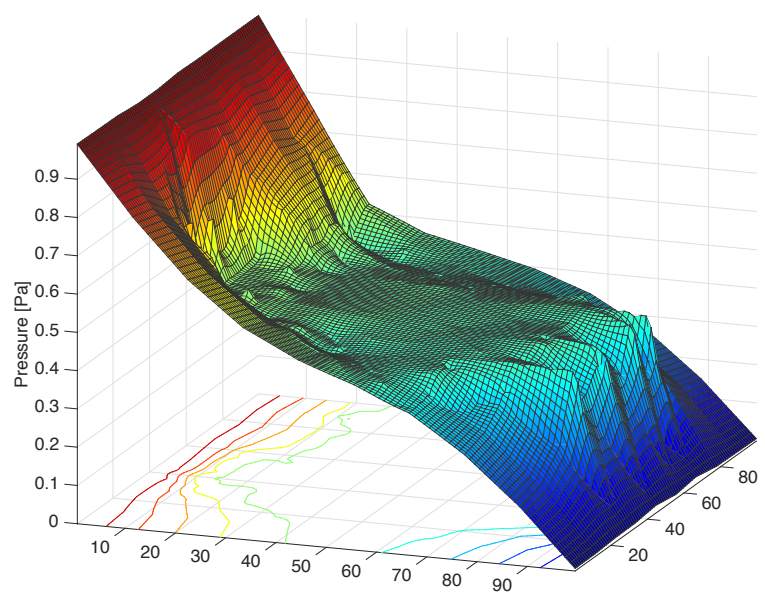

(c) F-MsRSB - 5 DOF in fracture $\left(\epsilon_{p}=2.02 \times 10^{-3}\right)$

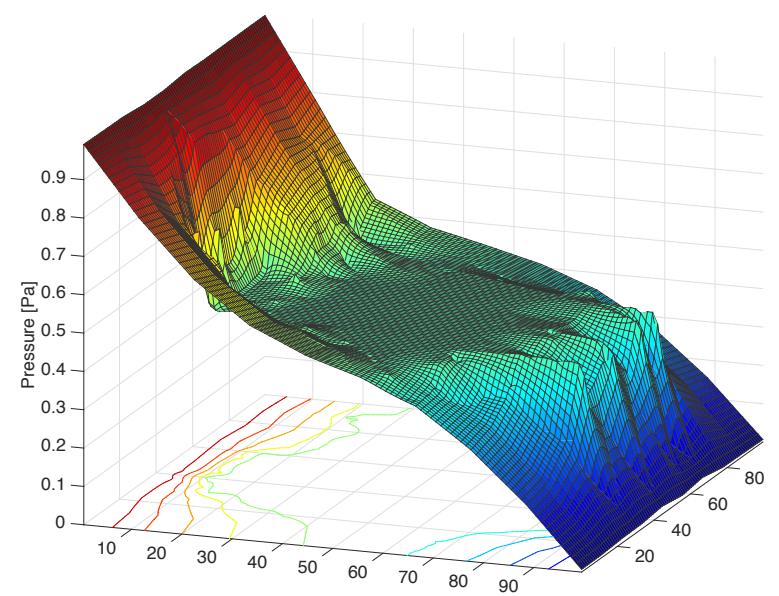

(b) F-MsRSB - 1 DOF in fracture

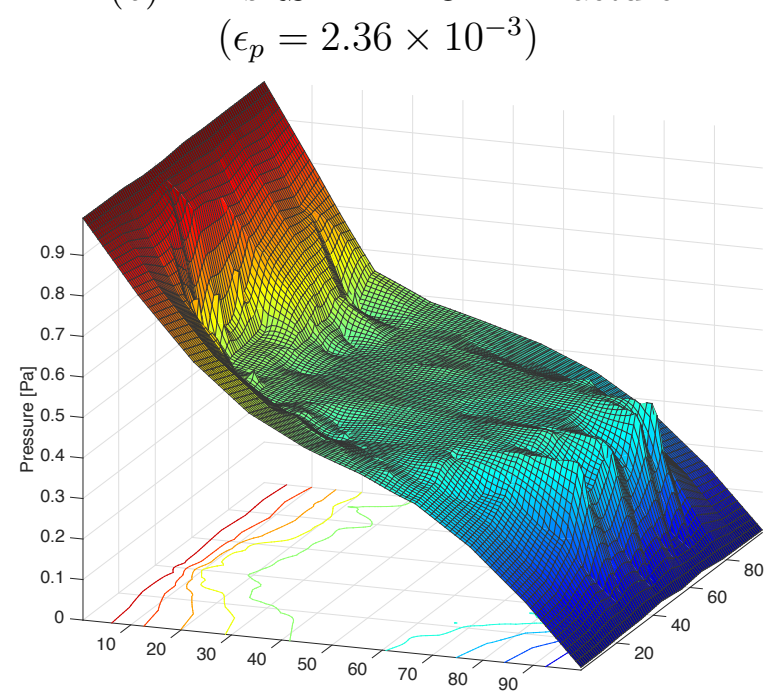

(d) F-MsRSB - 10 DOF in fracture $\left(\epsilon_{p}=1.67 \times 10^{-3}\right)$

Fig. 5.3: Reference and F-MsRSB pressure for the first test case as shown in Fig. 5.1. F-MsRSB solutions are presented for different fracture coarsening ratios.

\section{Outcrop fracture map}

The fracture coordinates are scaled from an outcrop photo [69] to fit a domain of size $1000 m \times 1000 m$, as shown in Fig. 5.5a. The fine-scale grid contains $100 \times 100$ matrix and 2074 fracture cells (over 94 disconnected fracture networks). The F-MsRSB grid contains $15 \times 15$ matrix and 155 fracture coarse cells. Fig. 5.5b shows the permeability of the matrix formation. Fracture permeability is set to 1000 Darcy.

We consider two-phase flow, with quadratic relative permeability curves and unit viscosity ratio between the phases. Fluid is injected at a constant rate in cell $(1,100)$, while fluids are produced in the opposite corner $(100,1)$ at constant pressure. Fig. 5.6 shows the saturation maps obtained after one F-MsRSB cycle (no iterations) compared with reference fine-scale solutions, both obtained using a sequentially-implicit strategy. 


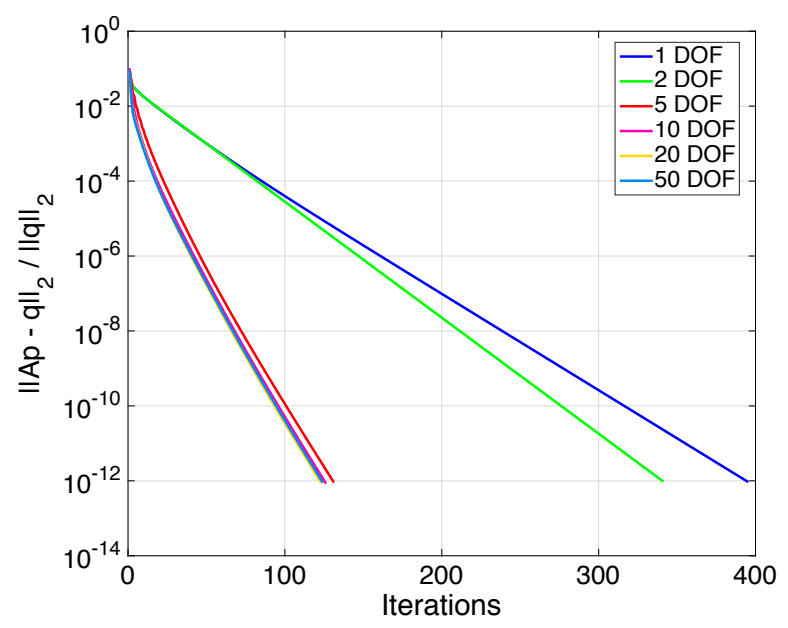

Fig. 5.4: Convergence of F-MsRSB+ILU(0) for different DOF in fracture.

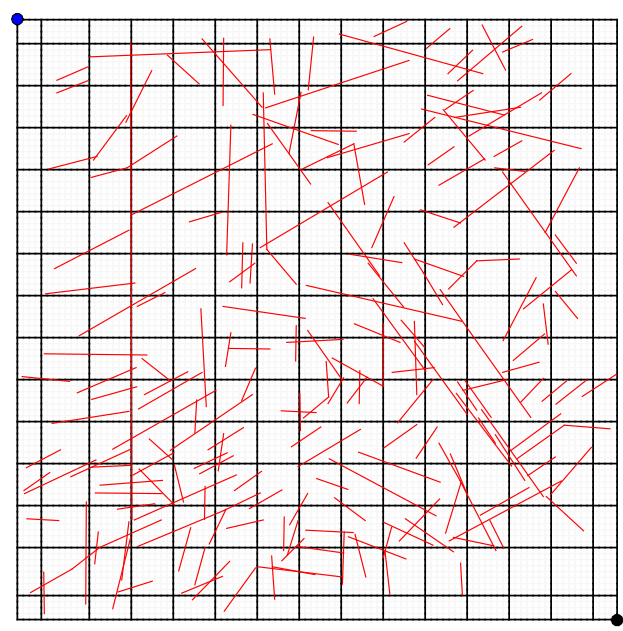

(a)

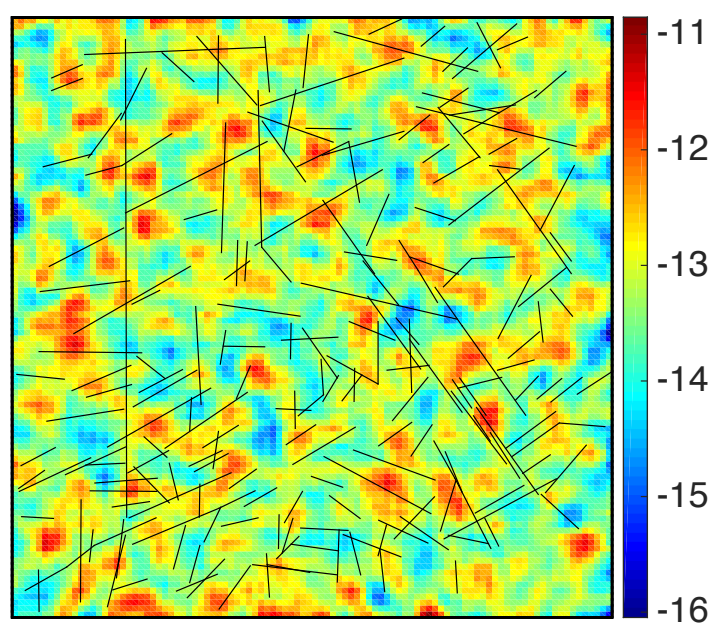

(b) $\log _{10}\left(k^{m}\right)\left[\mathrm{m}^{2}\right]$

Fig. 5.5: Matrix coarse grid showing well locations and fracture map extracted from an outcrop (a); Logarithm of the corresponding permeability field in the matrix (b).

Clearly, the higher the resolution of fracture coarse grid, the more accurate the FMsRSB results.

Saturation error is calculated as

$$
\epsilon_{S}=\frac{\max _{i \in n_{f}}|| S_{i}^{f s}-S_{i}^{m s}\left|\times \Omega_{i} \phi_{i}\right|}{\max _{i \in n_{f}}|| S_{i}^{f s}\left|\times \Omega_{i} \phi_{i}\right|},
$$

which, because it is scaled with pore volume, gives a very strict measure of the error in the spatial mass distribution for incompressible fluids.

As mentioned before, multiscale solutions can be improved by increasing the number of degrees of freedom per fracture network or by applying iterations (in combination with $\operatorname{ILU}(0)$, similar to $[1,2,58])$. Fig. 5.7b reports the saturation calculated at the producer grid block whereas Fig. 5.7b shows overall saturation errors. After only a few iterations of the two stage (F-MsRSB + ILU(0)) cycle, the multiscale fluid dis- 


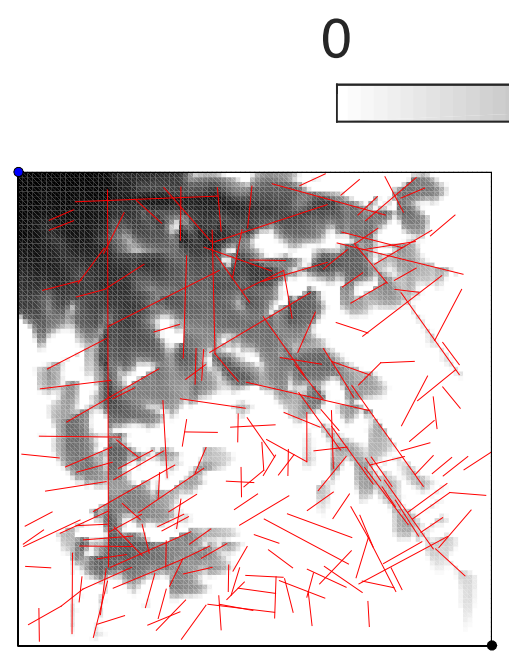

(a) Finescale: 0.25 PVI

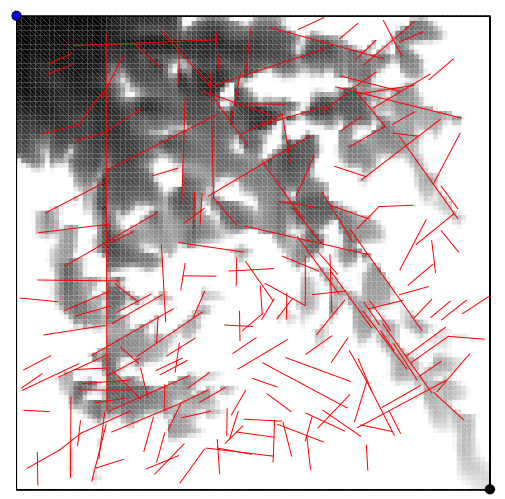

(d) F-MsRSB: 0.25 PVI

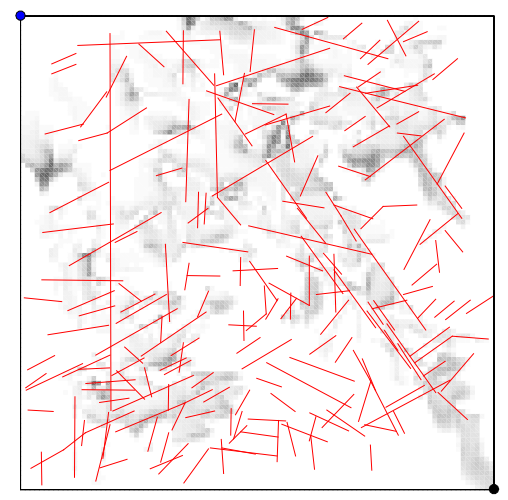

(g) $\left|S^{F-M s R S B}-S^{f s}\right|$

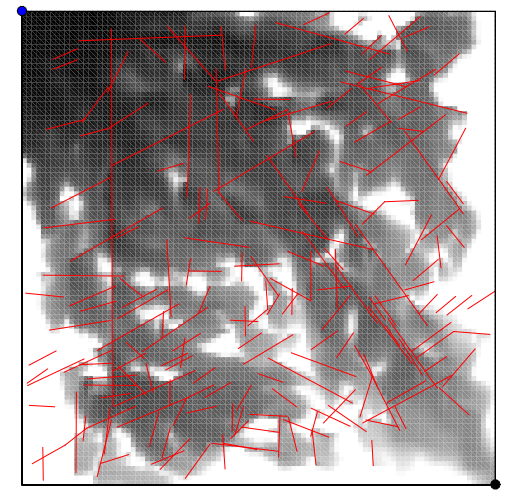

(b) Finescale: 0.50 PVI

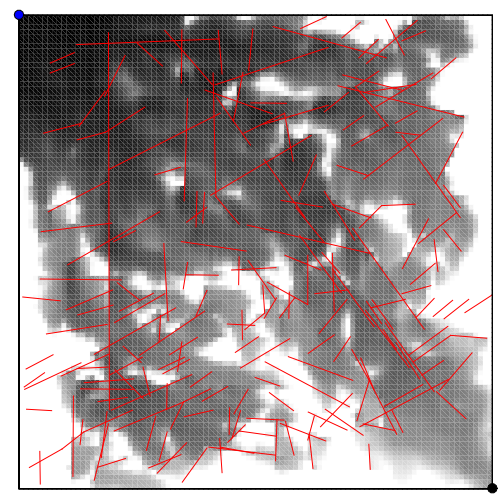

(e) F-MsRSB: 0.50 PVI

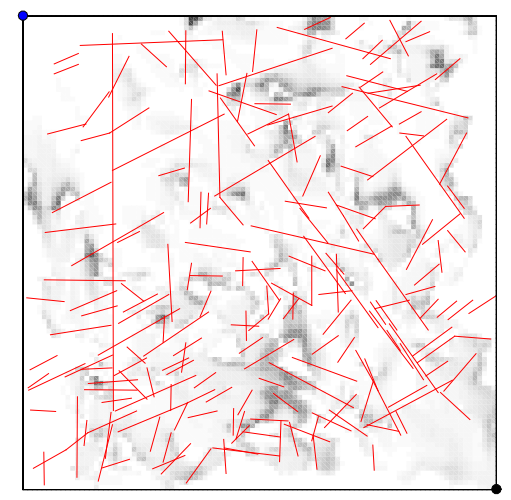

(h) $\left|S^{F-M s R S B}-S^{f s}\right|$

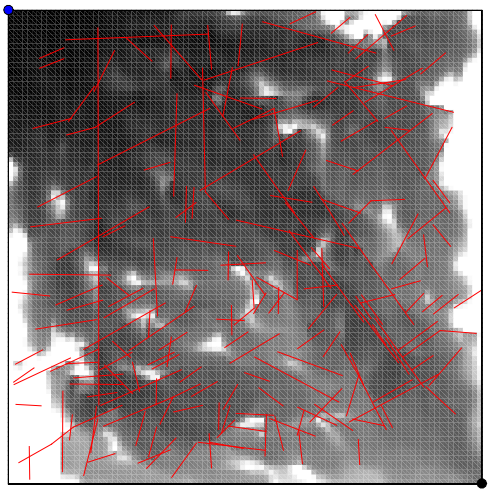

(c) Finescale: 0.75 PVI

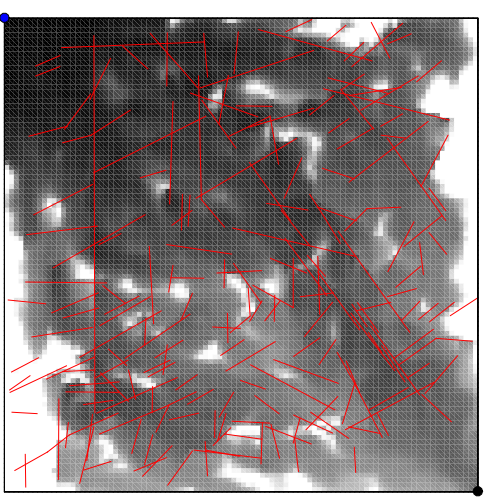

(f) F-MsRSB: 0.75 PVI

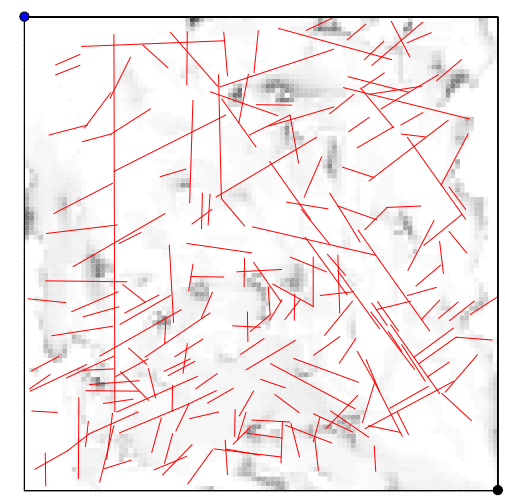

(i) $\left|S^{F-M s R S B}-S^{f s}\right|$

Fig. 5.6: Reference saturation profile compared with saturation maps obtained after one F-MsRSB cycle at different PV injected. Absolute errors in saturation are also shown in $(\mathrm{g})-(\mathbf{i})$.

tribution is virtually identical to the reference solution. Convergence to a tolerance of 0.1 takes 8 iterations for this outcrop model whereas a tolerance of 0.01 is reached after 20 iterations. One can also employ a local block solver around the fractures and wells [12]. 


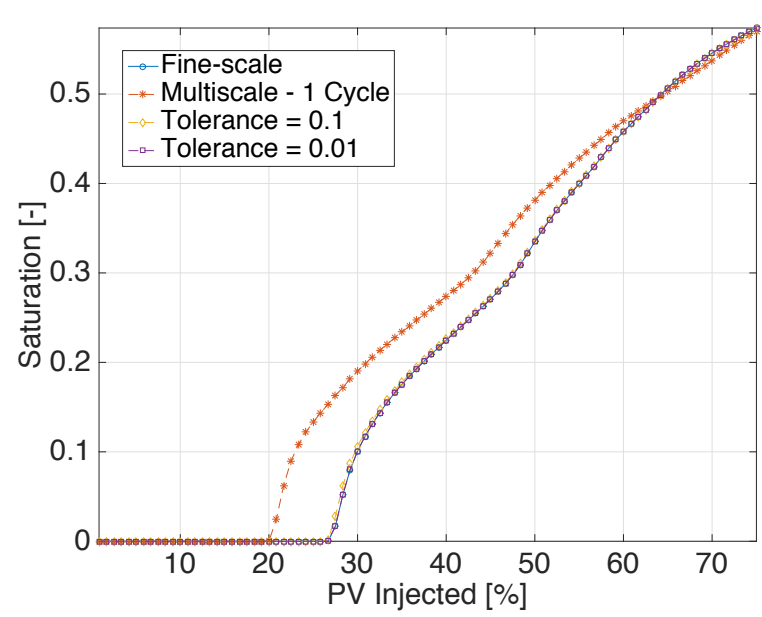

(a)

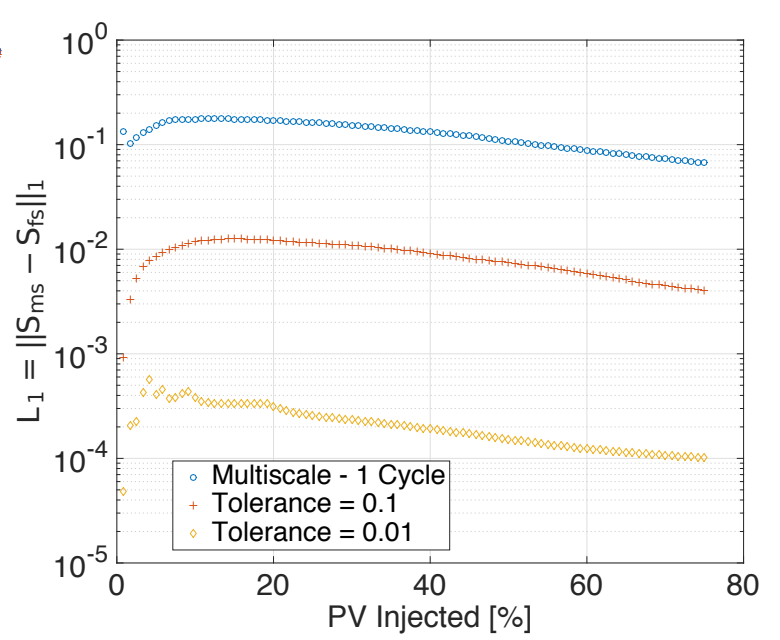

(b)

Fig. 5.7: Saturation at producer grid-block (a) and global saturation error (b) for outcrop model as a function of simulation time measured in pore-volume-injection. Shown are the corresponding results for non-iterative F-MsRSB and iterative F-MsRSB with the tolerances of 0.1 and 0.01 on the pressure solves.

\section{Statistical fracture model}

A $1000 m \times 500 m$ heterogeneous domain with permeability and porosity sampled from the 10-th layer of the SPE10 dataset [56] is considered and shown in Fig. 5.8. Similar as in previous test case, fracture permeability and porosity are 1000 Darcy and 0.50 , respectively. PEBI grids are employed for the matrix at fine scale, which is a voronoi map over a uniform triangulation in the region. Fine-scale grid contains 4726 cells for matrix and 2207 cells for fractures. There exist 55 disconnected fracture networks in the domain. Both matrix and fracture are coarsened using METIS [57] to give 100 coarse cells for each domain (fracture and matrix) as shown in Fig. 5.8a.

Incompressible oil and water phases with quadratic relative permeabilities are considered. The reservoir is initially filled with oil having a viscosity of $5 \mathrm{cP}$. Water with a lower viscosity of $1 \mathrm{cP}$ is injected from a well near the bottom left corner at a constant rate. Production occurs at constant pressure near the top right corner of the domain. Fig. 5.9 shows the saturation maps after 1 cycle of (F-MsRSB + ILU(0)) for injection amounts of $0.2,1.0$ and 1.8 pore volumes (PVI). The initial multiscale solution is already quite accurate, and after 1 smoothing-iteration step the multiscale and reference solutions are virtually identical. Fig. $\mathbf{5 . 1 0}$ compares bottom-hole pressure in the injector and oil rate in the producer as computed by the fine-scale reference solver and F-MsRSB for different iterative tolerances in the pressure solves.

\section{$5.3 \quad 3 \mathrm{D}$ models}

In this section, we study the performance of F-MsRSB for three examples in which the matrix domain is described in $3 \mathrm{D}$ and fractures are planar $2 \mathrm{D}$ surfaces. 


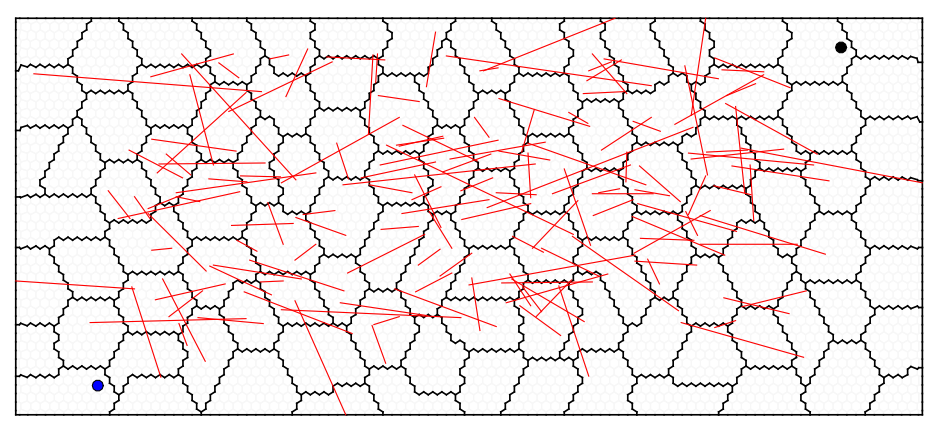

(a) Coarse grid with well locations

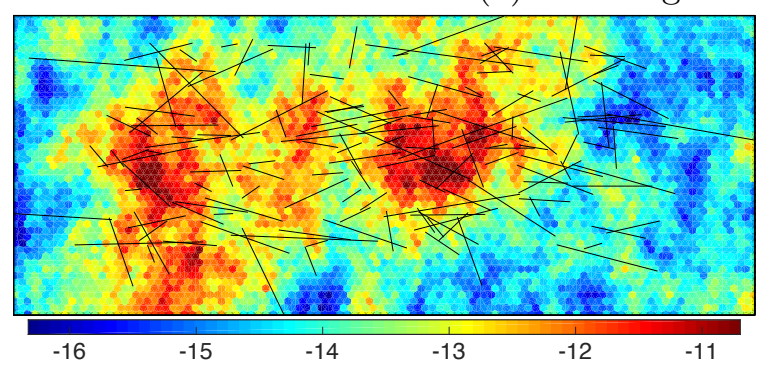

(b) $\log _{10}\left(k^{m}\right)\left[\mathrm{m}^{2}\right]$

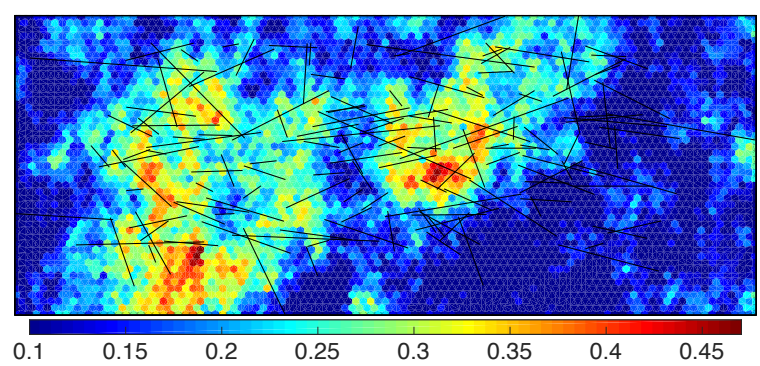

(c) $\phi^{m}$

Fig. 5.8: Matrix coarse grid with 100 DOF, fracture map and well locations (a); Petrophysical rock properties sampled from the $10^{\text {th }}$ layer of the tarbert formation in the SPE10 dataset (b and $\mathbf{c})$.

\section{Two intersecting fracture planes}

The fine grid for the first $3 \mathrm{D}$ example consists of $50 \times 50 \times 50$ matrix cells and 2 fracture plates, each with $100 \times 30$ fracture grid cells. The fracture plates cross in the middle of the domain, as shown in Fig. 5.11a. Injection occurs at a constant rate at the bottom-left corner, while production takes place from the top-right corner, both through horizontal wells. Matrix permeability is shown in Fig. 5.11b. The F-MsRSB grid contains $20 \times 20 \times 20$ matrix blocks and $12 \times 4$ (for each plate) fracture blocks. Fracture permeability is set to $10^{4}$ Darcy. The matrix coarsening ratio is chosen such that the effect of fracture coarsening ratios will be more pronounced in the F-MsRSB results. Fig. 5.12 shows pressure solution obtained after 1 F-MsRSB step. In addition, Fig. 5.13 presents the convergence behaviour for different coarse grid resolutions for fracture plates. As for the small matrix coarsening ratio, it is observed that small increase in number of fracture DOF leads to significantly improved convergence rates.

\section{Depositional bed model}

As another 3D example, a corner-point grid model is considered with a wavy depositional bed and several degenerate cells. A similar model has been used in the literature [11]. Pinch-outs, occurring mainly due to the accounting of erosional features, are a common reason behind unstructured cell connections in stratigraphic corner-point grids. They lead to degenerate cells with faces of zero area resulting in a complex grid geometry. With the addition of heterogeneity, it becomes quite a challenging test case for multiscale methods $[14,21]$. In order to improve the efficiency of F-MsRSB preprocessing steps (such as computing fracture-matrix transmissibility) for this challenging grid geometry, first the $C I$ factors (Eq. (2.8)) are calculated globally for each fracture 


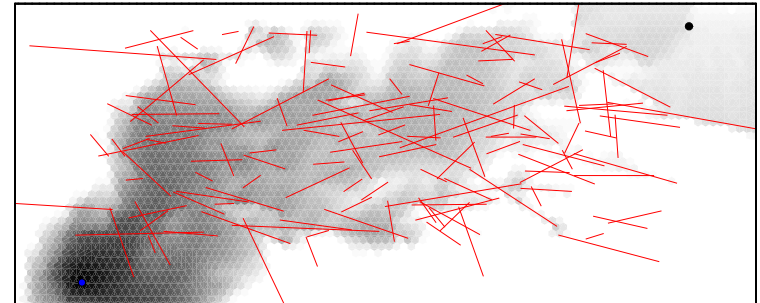

(a) Finescale: 0.2 PVI

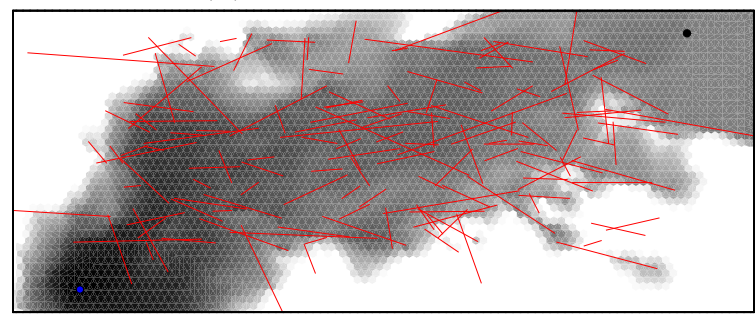

(c) Finescale: 1.0 PVI

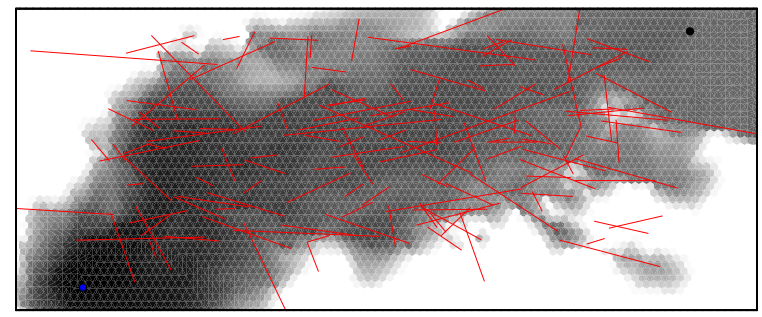

(e) Finescale: 1.8 PVI

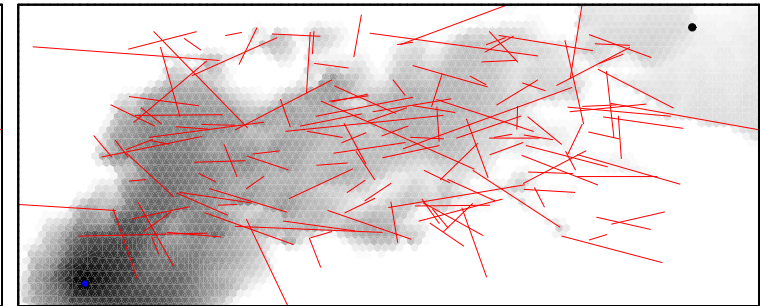

(b) F-MsRSB: 0.2 PVI

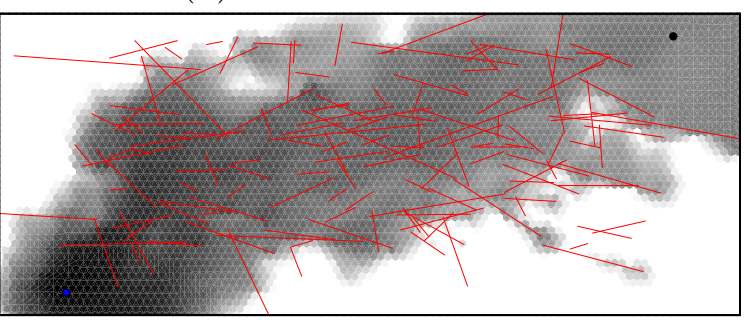

(d) F-MsRSB: 1.0 PVI

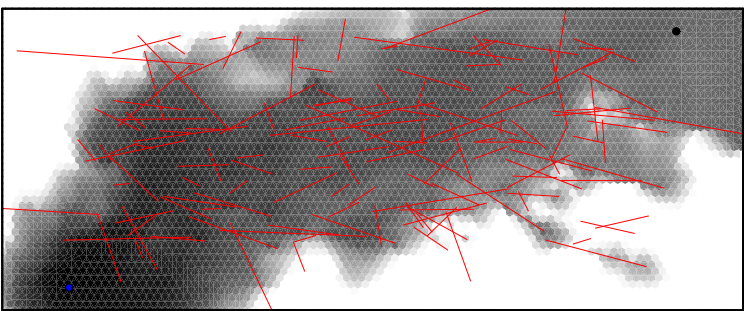

(f) F-MsRSB: 1.8 PVI

Fig. 5.9: Saturation maps for fine-scale reference and F-MsRSB after 1 cycle of iteration (F-MsRSB + ILU(0)) at different simulation times.

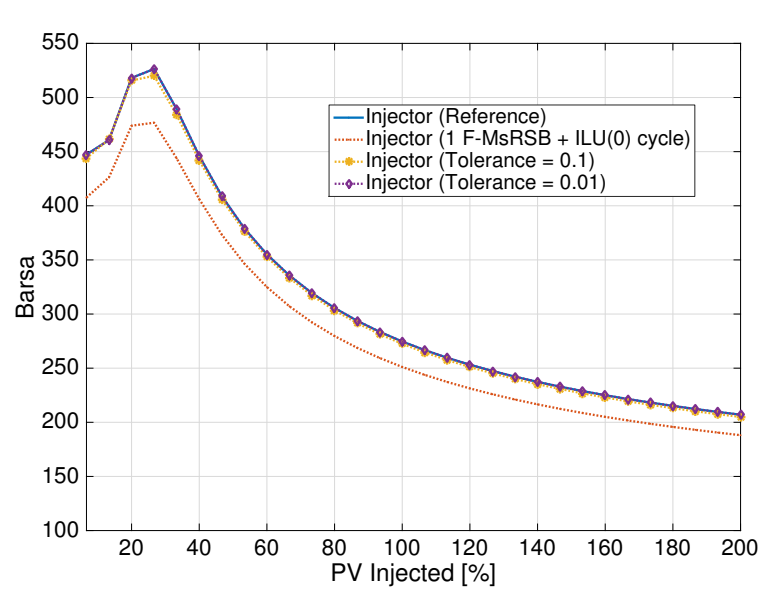

(a) Injector bottom hole pressure

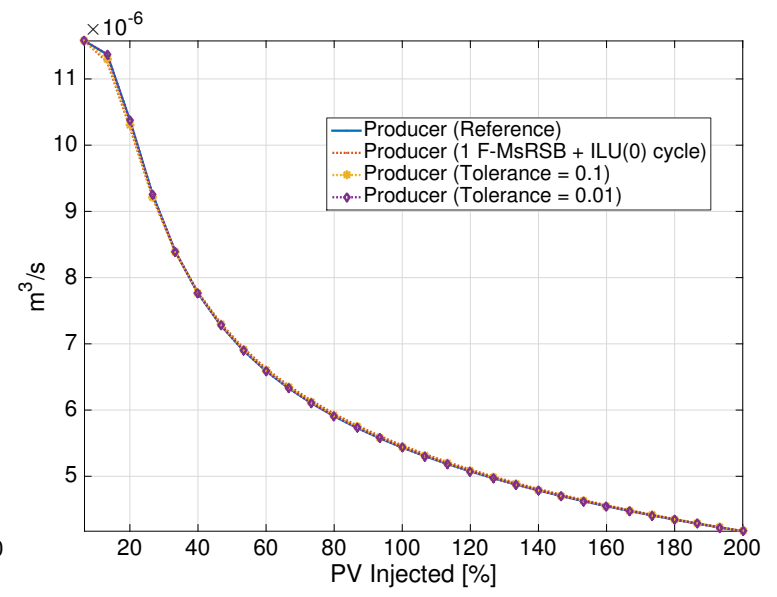

(b) Oil rate at producer

Fig. 5.10: Production and injection quantities at well locations for the statistical 2D fracture map.

plane. Then, they're computed for each fracture-matrix overlapping discrete grid cell.

The fine-scale grid contains $30 \times 30 \times 100$ matrix with 222 hexahedral fracture cells per fracture plate. As shown in Fig. 5.14a, there exist 6 fracture plates, and the domain is subject to Dirichlet boundary conditions on the left and right faces, while 


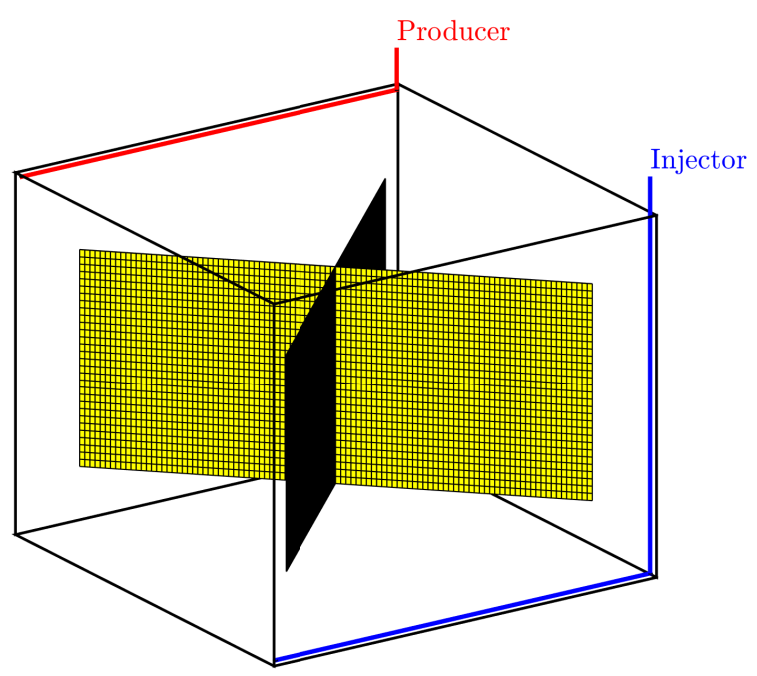

(a) Fracture planes and wells.

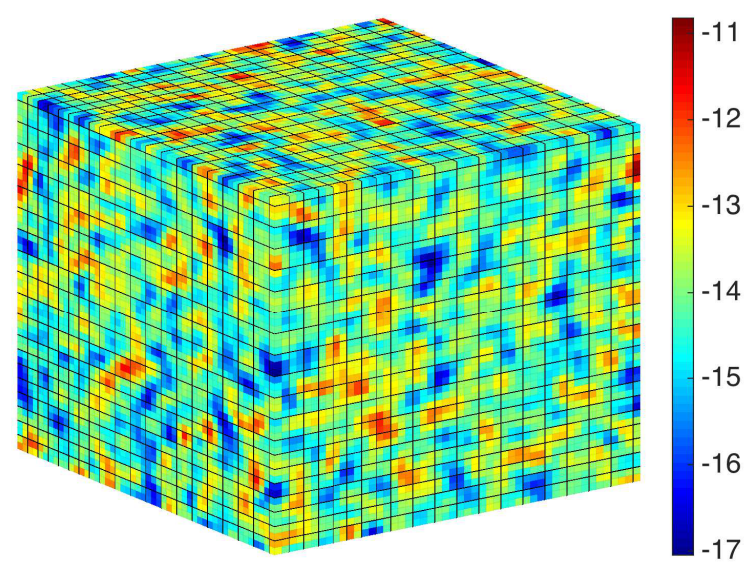

(b) $\log _{10}\left(k^{m}\right)\left[\mathrm{m}^{2}\right]$ and F-MsRSB coarse partition.

Fig. 5.11: Illustration of the first 3D case, with a fine-scale grid that contains $50 \times 50 \times$ 50 matrix cells and two intersecting fracture planes that each contain $100 \times 30$ fracture cells. Also shown on the right is heterogeneous matrix permeability map, along with the imposed $20 \times 20 \times 20$ coarse grid used by F-MsRSB.

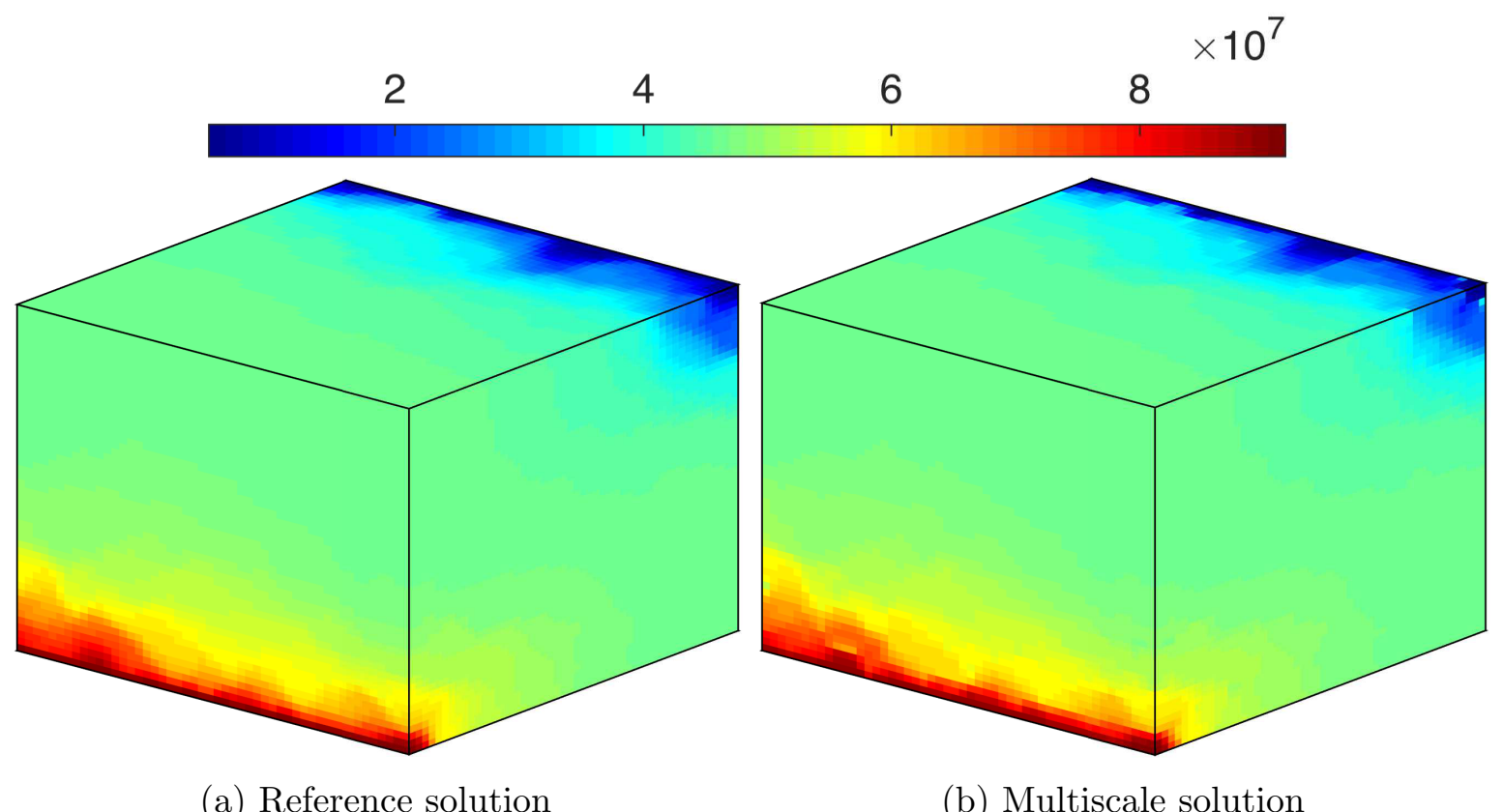

Fig. 5.12: Reference and multiscale pressure solution after $1 \mathrm{~F}$-MsRSB cycle for single phase flow in the simple 3D model. Each fracture plane is logically partitioned into $12 \times 4$ blocks.

all other faces are subject to no-flow condition. As shown in Fig. 5.14, F-MsRSB employs $10 \times 10 \times 9$ coarse cells for matrix, and only 2 coarse cells for each fracture plate. In addition, fracture aperture is $0.04 \mathrm{~m}$. The matrix permeability distribution is provided in Fig. 5.14b, and $k_{f}=10000 \mathrm{D}$, resulting in quite large contrasts in the permeability values throughout the entire model. 


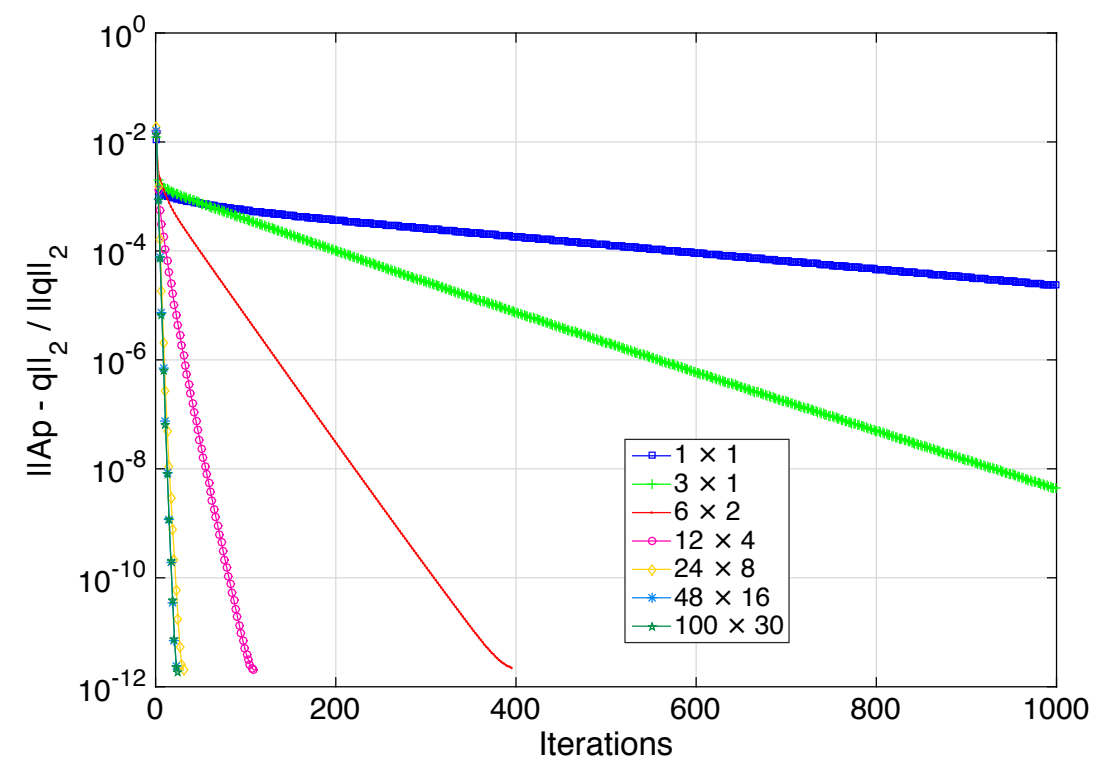

Fig. 5.13: Convergence property of the F-MsRSB + ILU (0) solver for various coarse grid resolutions per fracture plane. Each fracture plane is of size $100 \times 30$ at fine scale.

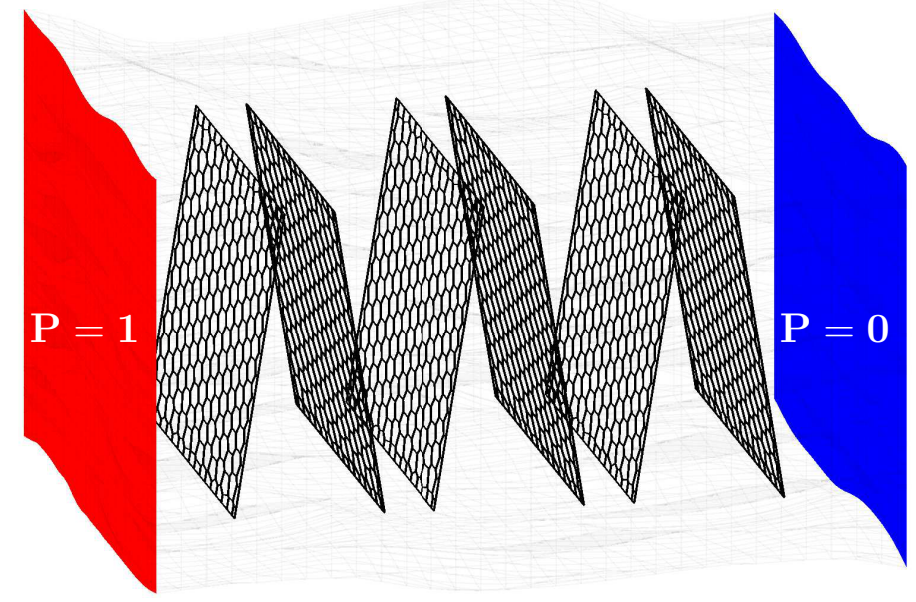

(a) Model outline, fractures, and boundary conditions

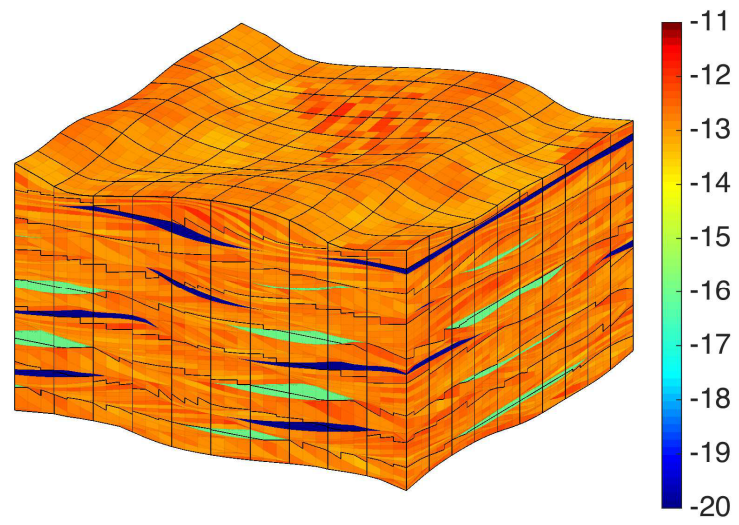

(b) $\log _{10}\left(k^{m}\right)\left[\mathrm{m}^{2}\right]$

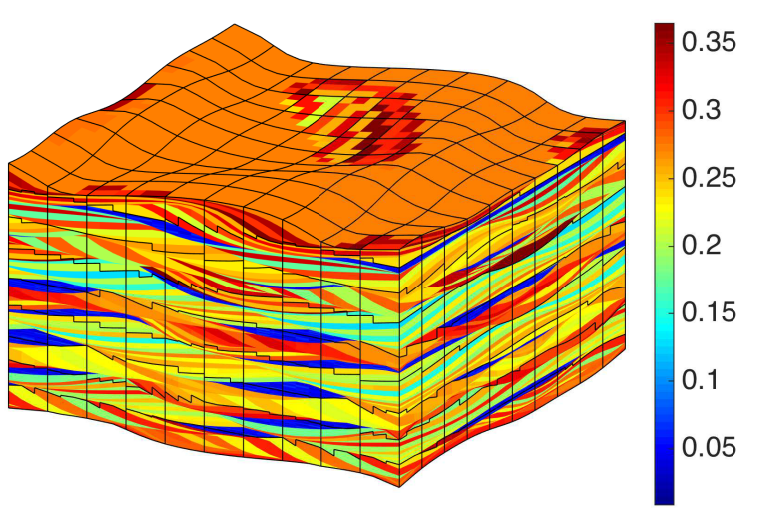

(c) $\phi^{m}$

Fig. 5.14: Matrix grid with fracture planes and boundary conditions (a); Logarithm of permeability map (b) and matrix porosity (c) 
Fig. 5.15 shows pressure solutions obtained after 1 F-MsRSB step. It is clear that F-MsRSB and reference solutions are in good agreement, even with such a large coarsening ratio for fractures. The absolute difference between the two solutions is depicted in Fig. 5.15c, with the pressure error $\left(\epsilon_{p}\right)$ being $8.79 \times 10^{-4}$.

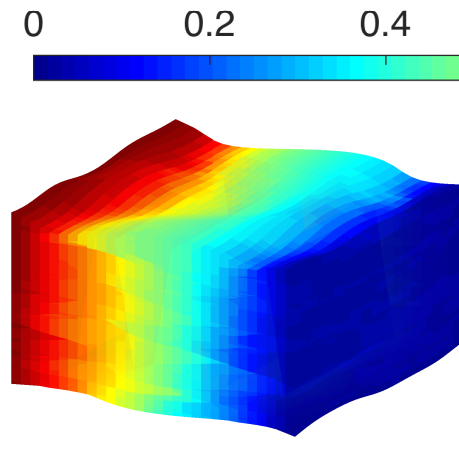

(a) Reference

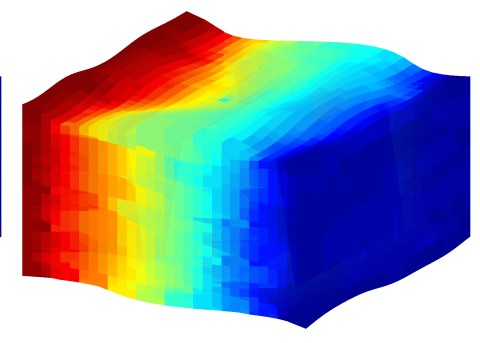

(b) F-MsRSB

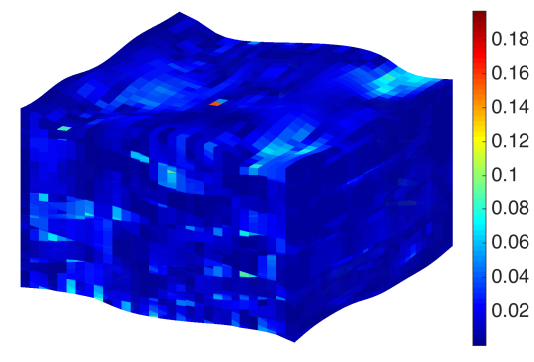

(c) $\left|p^{m s}-p^{f s}\right|$

Fig. 5.15: Reference and multiscale pressure solution for single phase flow in the bed model with 2 degrees of freedom per fracture plane.

\section{Reduced Model 2 of SPE10 with fracture networks}

As the final test case in this section, the challenging SPE10 permeability is extracted from the full model (which contains $60 \times 220 \times 85$ fine cells) for a fine-scale grid size of $30 \times 110 \times 40$ [56]. As shown in Fig. 5.16, complex fracture plates (located between layers 11 through 30 ) are obtained by extruding the statistical maps, similar to the ones used for one of the 2D test cases. The model contains 31 disconnected fracture networks, which are discretized using 13, 880 fine-scale grid cells.

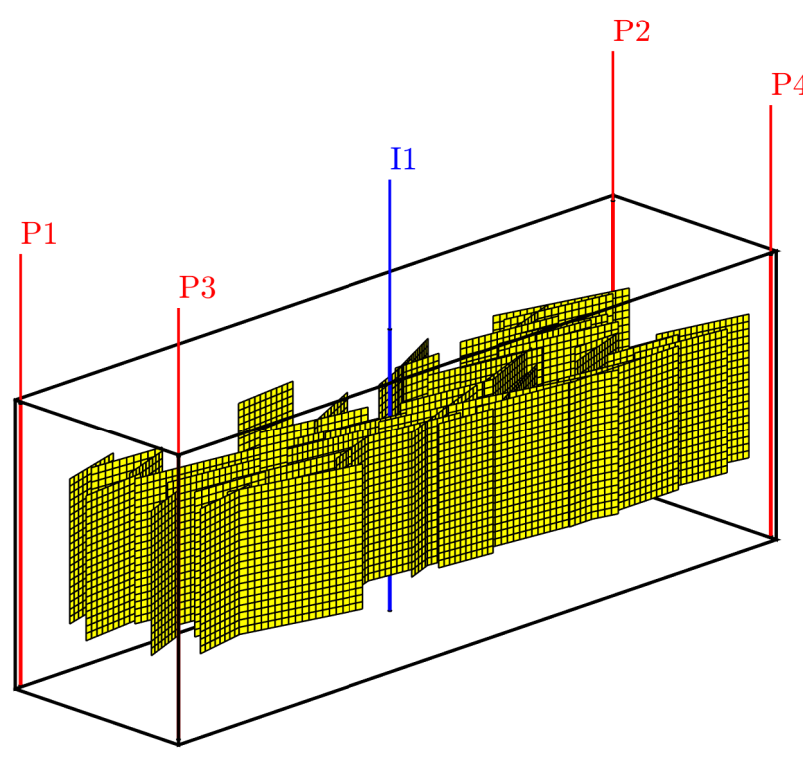

(a) 3D Test Case 3

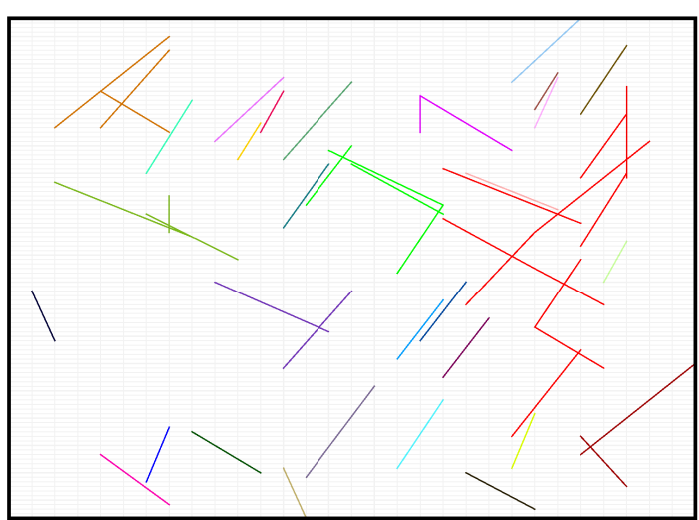

(b) Scaled top view.

Fig. 5.16: 5-spot well locations in the 3D Test Case 3, with 31 disconnected fracture networks. Shown on the right is the top view of the model. 
Fig. 5.17 shows the matrix rock properties (permeability and porosity). All fractures have permeability value of 1000 Darcy. F-MsRSB grids contain $6 \times 22 \times 8$ matrix and 181 fracture coarse cells in total. Each fracture coarse cell contains 80 fine-scale fracture cells on average.

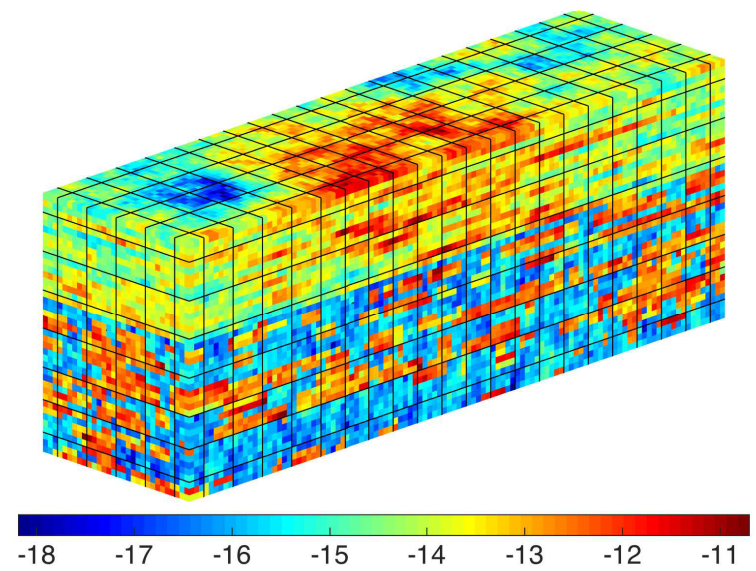

(a) $\log _{10}\left(k^{m}\right)\left[\mathrm{m}^{2}\right]$

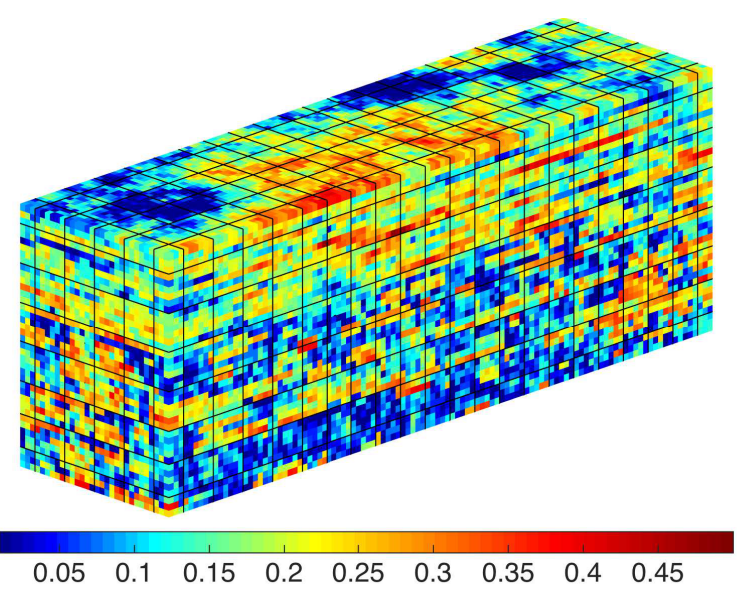

(b) $\phi^{m}$

Fig. 5.17: Petrophysical properties for the $30 \times 110 \times 40$ domain sampled from the full SPE10 dataset

A waterflood experiment has been considered for the duration of 5 PVI, using quadratic relative permeability values. Water of viscosity $1 \mathrm{cP}$ is injected into the reservoir which is initially filled with $100 \%$ oil of viscosity $10 \mathrm{cP}$. As shown in Fig. 5.16a, five wells are placed in a 5-spot pattern with a fixed rate injector in the middle and 4 fixed-pressure producers at the corners.

F-MsRSB results are presented in Fig. $\mathbf{5 . 1 8}$ at the location of injection and production, compared with reference fine-scale solutions. As shown, one iteration of (FMsRSB+ILU(0)) leads to significantly improved solutions. The initial multiscale solution residual is approximately 0.1 , which reduces to approximately 0.01 after only one smoothing iteration. Convergence to a tolerance of $10^{-3}$ and subsequently to $10^{-4}$ takes around 5 and 15 average iterations, respectively, per time step. 


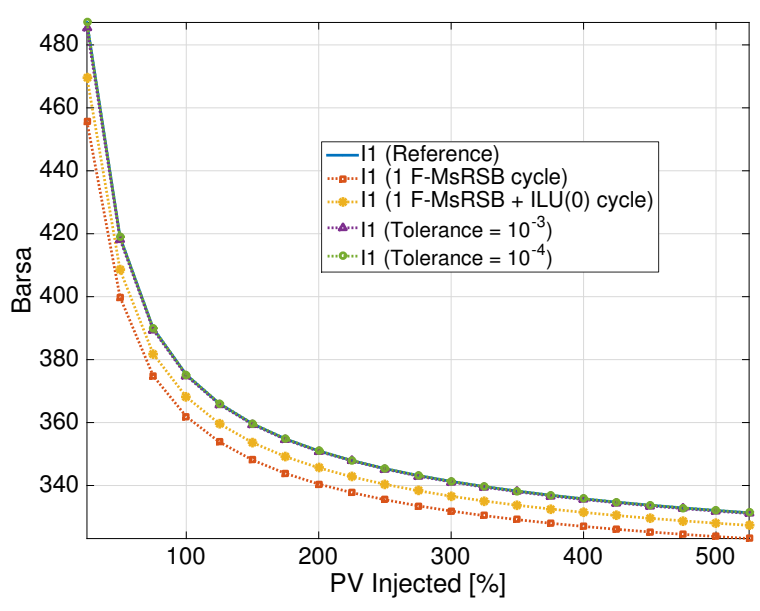

(a) Injector bottom hole pressure

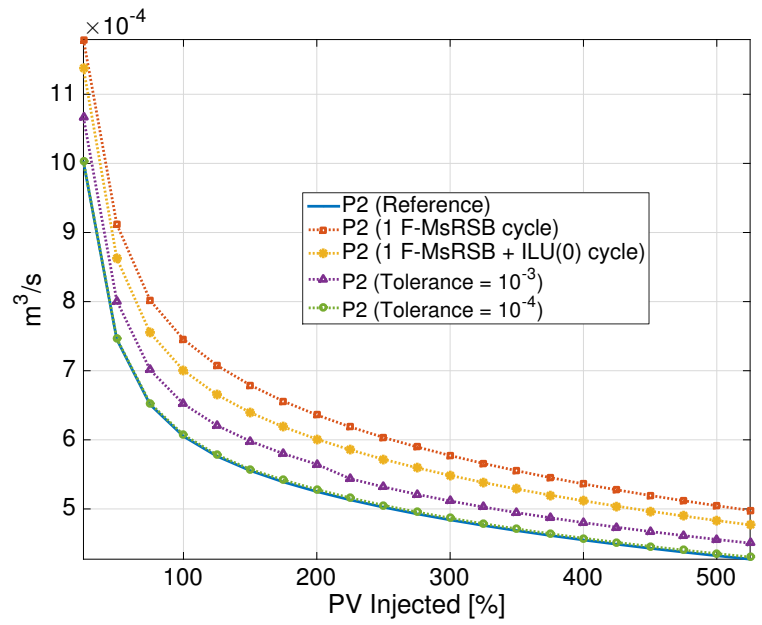

(c) Oil rate at producer $\mathrm{P} 2$

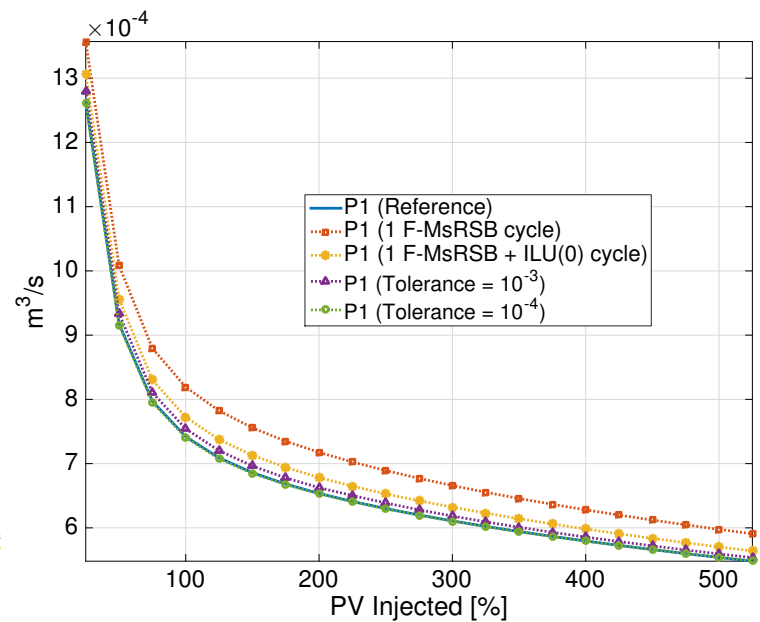

(b) Oil rate at producer $\mathrm{P} 1$

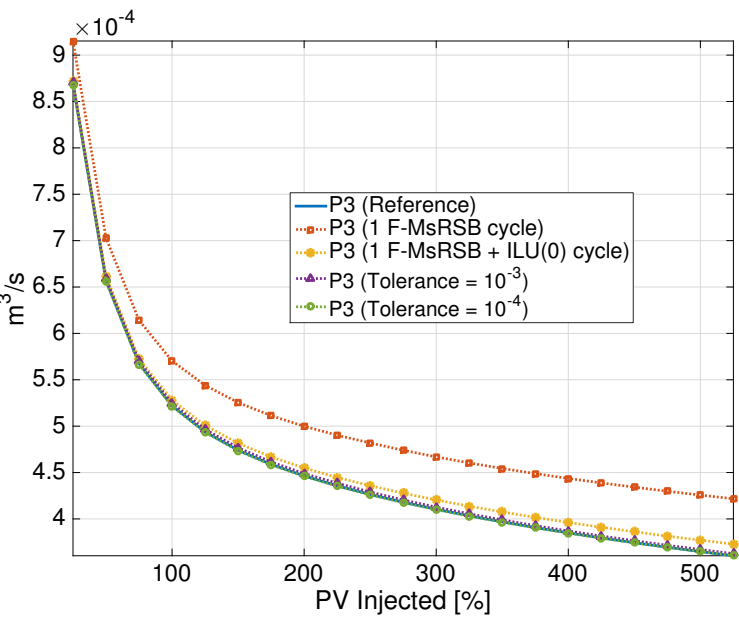

(d) Oil rate at producer $\mathrm{P} 3$

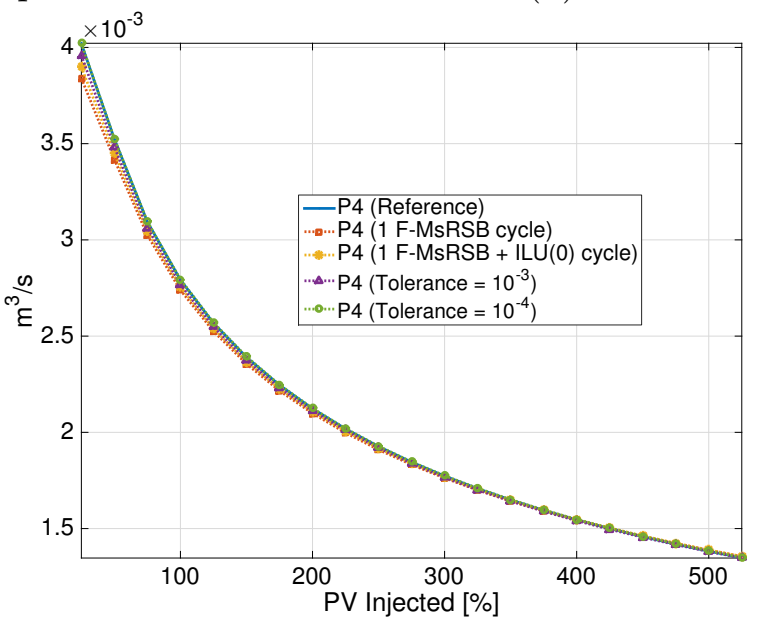

(e) Oil rate at producer $\mathrm{P} 4$

Fig. 5.18: Solution at the location of wells for the 3D Test Case 3. F-MsRSB solutions are shown for different tolerances of pressure solution, compared with reference solutions. 


\section{Chapter 6}

\section{Conclusion}

In this thesis, a novel multiscale framework for fractured porous media (F-MsRSB) was introduced. The method benefited from the most recent developments within the multiscale community, namely F-AMS and MsRSB, yet devised a unique approach for robust and efficient treatment of fractured media. Efficiency and accuracy of the devised multiscale method, F-MsRSB, was analysed for many challenging test cases, including a realistic fracture map extracted from an outcrop. These extensive studies were quite unique in the multiscale community. F-MsRSB is formulated and implemented in an algebraic form into the open-source MATLAB Reservoir Simulation toolbox, MRST (Sintef ICT, Dept. Applied Mathematics). This development, thus, is open to the public scientific community; another important contribution of this work.

Through a set of single- and multiphase test cases it was found that F-MsRSB can efficiently simulate models of fractured porous media with highly heterogeneous coefficients and produce approximate solutions with a prescribed fine-scale residual accuracy. The numerical test cases also included complex wells. By using an adaptive iterative strategy, one can trade accuracy for computational efficiency, and still produce mass-conservative, approximate solutions on the fine scale.

Ongoing research includes consideration of more challenging fluid and rock physics, along with integration of F-MsRSB into the in-house $\mathrm{C}++$ simulator for comparisons of CPU efficiency. 


\section{References}

[1] H. Hajibeygi, D. Karvounis, and P. Jenny. A hierarchical fracture model for the iterative multiscale finite volume method. J. Comput. Phys., 230(24):8729-8743, 2011.

[2] M. Tene, M. S. Al Kobaisi, and H. Hajibeygi. Algebraic multiscale solver for flow in heterogeneous fractured porous media. In SPE Reservoir Simulation Symposium, 2015.

[3] O. Møyner and K.-A. Lie. A multiscale method based on restriction-smoothed basis functions suitable for general grids in high contrast media. In SPE Reservoir Simulation Symposium, 2015.

[4] K. Aziz and A. Settari. Petroleum Reservoir Simulation. Blitzprint Ltd., Cagary, Alberta, 2002.

[5] T. Y. Hou and X.-H. Wu. A multiscale finite element method for elliptic problems in composite materials and porous media. J. Comput. Phys., 134(1):169-189, 1997.

[6] P. Jenny, S. H. Lee, and H. A. Tchelepi. Multi-scale finite-volume method for elliptic problems in subsurface flow simulation. J. Comput. Phys., 187(1):47-67, 2003.

[7] P. Jenny, S. H. Lee, and H. A. Tchelepi. Adaptive fully implicit multi-scale finitevolume method for multi-phase flow and transport in heterogeneous porous media. J. Comput. Phys., 217(2):627-641, 2006.

[8] Y. Efendiev and T. Y. Hou. Multiscale Finite Element Methods: Theory and Applications, volume 4. Springer Science \& Business Media, 2009.

[9] V. Kippe, J. E. Aarnes, and K.-A. Lie. A comparison of multiscale methods for elliptic problems in porous media flow. Comput. Geosci., 12(3):377-398, 2008.

[10] J. E. Aarnes, V. Kippe, and K.-A. Lie. Mixed multiscale finite elements and streamline methods for reservoir simulation of large geomodels. Adv. Water Resour., 28(3):257-271, 2005.

[11] J. E. Aarnes, S. Krogstad, and K.-A. Lie. Multiscale mixed/mimetic methods on corner-point grids. Comput. Geosci., 12(3):297-315, 2008.

[12] H. Hajibeygi and P. Jenny. Adaptive iterative multiscale finite volume method. J. Comput. Phys., 230(3):628-643, 2011. 
[13] I. Lunati and P. Jenny. Multiscale finite-volume method for density-driven flow in porous media. Comput. Geosci., 12(3):337-350, 2008.

[14] O. Møyner and K.-A. Lie. A multiscale two-point flux-approximation method. $J$. Comput. Phys., 275:273-293, 2014.

[15] H. Hajibeygi, R. Deb, and P. Jenny. Multiscale finite volume method for nonconformal coarse grids arising from faulted porous media. In SPE Reservoir Simulation Symposium, 2011.

[16] Y. Wang, H. Hajibeygi, and H. A. Tchelepi. Monotone multiscale finite volume method. Accepted, in-press, Comput. Geosci., 2015.

[17] H. Hajibeygi and P. Jenny. Multiscale finite-volume method for parabolic problems arising from compressible multiphase flow in porous media. J. Comput. Phys., 228(14):5129-5147, 2009.

[18] H. Zhou and H. A. Tchelepi. Operator based multiscale method for compressible flow. SPE J., 13(2):267-273, 2008.

[19] P. Jenny and I. Lunati. Modeling complex wells with the multi-scale finite volume method. J. Comput. Phys., 228(3):687-702, 2009.

[20] S. H. Lee, H. Zhou, and H. Techelpi. Adaptive multiscale finite-volume method for nonlinear multiphase transport in heterogeneous formations. J. Comput. Phys., 228(24):9036-9058, 2009.

[21] O. Møyner and K.-A. Lie. The multiscale finite-volume method on stratigraphic grids. SPE J., 19(05):816-831, 2014.

[22] H. Hajibeygi, S. H. Lee, and I. Lunati. Accurate and efficient simulation of multiphase flow in a heterogeneous reservoir by using error estimate and control in the multiscale finite-volume framework. SPE J., 17(4):1071-1083, 2012.

[23] H. Hajibeygi, G. Bonfigli, M. A. Hesse, and P. Jenny. Iterative multiscale finitevolume method. J. Comput. Phys., 227(19):8604-8621, 2008.

[24] H. Zhou and H. A. Tchelepi. Two-stage algebraic multiscale linear solver for highly heterogeneous reservoir models. SPE J., 17(2):523-539, 2012.

[25] Y. Wang, H. Hajibeygi, and H. A. Tchelepi. Algebraic multiscale linear solver for heterogeneous elliptic problems. Journal of Computational Physics, 259:284-303, 2014 .

[26] M. Tene, Y. Wang, and H. Hajibeygi. Adaptive algebraic multiscale solver for compressible flow in heterogeneous porous media. J. Comp. Phys., 2015.

[27] U. Trottenberg, C.W. Oosterlee, and A. Schueller. Multigrid. Elsevier Academic Press, 2001.

[28] S. H. Lee, C. Wolfsteiner, and H. A. Tchelepi. Multiscale finite-volume formulation for multiphase flow in porous media: black oil formulation of compressible, threephase flow with gravity. Comput. Geosci., 12(3):351-366, 2008. 
[29] H. Hajibeygi and H. A. Tchelepi. Compositional multiscale finite-volume formulation. SPE J., 19(2):316-326, 2014.

[30] O. Møyner and K.-A. Lie. The multiscale finite volume method on unstructured grids. In SPE Reservoir Simulation Symposium, 2013.

[31] M. Cusini, A. Lukyanov, J. Natvig, and H. Hajibygi. Constrained pressure residual multiscale (CPR-MS) method for fully implicit simulation of multiphase flow in porous media. J. Comp. Phys., 299:472-486, 2015.

[32] G. Barenblatt, Y Zheltov, and I. Kochina. Basic concepts in the theory of seepage of homogeneous fluids in fissurized rocks. J. Appl. Math. Mech., 5(24):1286-1303, 1983.

[33] J. Warren and P. Root. The behavior of naturally fractured reservoirs. SPE J., 3(3):245-255, 1963.

[34] H. Kazemi. Pressure transient analysis of naturally fractured reservoirs with uniform fracture distribution. SPE J., 9(4):451-462, 1969.

[35] L. K. Thomas, T. N. Dixon, and R. G. Pierson. Fractured reservoir simulation. SPE J., 23(1):42-54, 1983.

[36] R. Baca, R. Arnett, and D. Langford. Modeling fluid flow in fractured porous rock masses by finite element techniques. Int. J. Num. Meth. Fluids, 4(4):337-348, 1984.

[37] S. H. Lee, M. F. Lough, and C. L. Jensen. Hierarchical modeling of flow in naturally fractured formations with multiple length scales. Water Resources Research, 37(3):443-455, 2001.

[38] S. H. Lee, C. L. Jensen, and M. F. Lough. Efficient finite-difference model for flow in a reservoir with multiple length-scale fractures. SPE J., 3(5):268-275, 2000.

[39] L. Li and S. H. Lee. Efficient field-scale simulation of black oil in naturally fractured reservoir through discrete fracture networks and homogenized media. SPE Res. Eval. Eng., 11(4):750-758, 2008.

[40] J. R. Natvig, B. Skaflestad, F. Bratvedt, K. Bratvedt, K.-A. Lie, V. Laptev, and S.K. Khataniar. Multiscale mimetic solvers for efficient streamline simulation of fractured reservoirs. SPE J., 16(4):880-888, 2009.

[41] A. F. Gulbransen, V. L. Hauge, and K.-A. Lie. A multiscale mixed finite element method for vuggy and naturally fractured reservoirs. SPE J., 15(2):395-403, 2010.

[42] T. Barkve and A. Firoozabadi. Analysis of reinfiltration in fractured porous media. In SPE Annual Technical Conference and Exhibition, Washington D.C., USA, 1992.

[43] J. Noorishad and M. Mehran. An upstream finite element method for solution of transient transport equation in fractured porous media. Water Resour. Res., 3(18):588-596, 1982. 
[44] M. Karimi-Fard and A. Firoozabadi. Numerical simulation of water injection in $2 \mathrm{~d}$ fractured media using discrete-fracture model. In SPE Annual Technical Conference and Exhibition, 2001.

[45] M. Karimi-Fard, L. J. Durlofsky, and K. Aziz. An efficient discrete-fracture model applicable for general-purpose reservoir simulators. SPE J., 9(2):227-236, 2004.

[46] A. Moinfar, A. Varavei, K. Sepehrnoori, and R. T. Johns. Development of a coupled dual continuum and discrete fracture model for the simulation of unconventional reservoirs. In SPE Reservoir Simulation Symposium, The Woodlands, Texas, USA, 2013.

[47] R. Ahmed, M.G. Edwards, S. Lamine, B.A.H. Huisman, and M. Pal. Controlvolume distributed multi-point flux approximation coupled with a lowerdimensional fracture model. J. Comput. Phys., 284(1):462 - 489, 2015.

[48] H. Hajibeygi. Iterative multiscale finite-volume method for multiphase flow in porous media with complex physics. Phd thesis, ETH Zurich, Switzerland, 2011.

[49] A. Moinfar, K. Sepehrnoori, R. T. Johns, and A. Varavei. Coupled geomechanics and flow simulation for an embedded discrete fracture model. In SPE Reservoir Simulation Symposium, The Woodlands, Texas, USA, 2013.

[50] I. S. Ligaarden, M. Krokiewski, K.-A. Lie, D. W. Schmid, and M. Pal. On the Stokes-Brinkman equations for modeling flow in carbonate reservoirs. In Proceedings of ECMOR XII-12th European Conference on the Mathematics of Oil Recovery, Oxford, UK, 2010. EAGE.

[51] T. H. Sandve, I. Berre, E. Keilegavlen, and J. Martin Nordbotten. Multiscale simulation of flow and heat transport in fractured geothermal reservoirs: inexact solvers and improved transport upscaling. In Thirty-Eighth Workshop on Geothermal Reservoir Engineering Stanford University, Stanford, California, USA, 2013.

[52] M. Tene, M. S. Al Kobaisi, and H. Hajibeygi. Algebraic multiscale solver for fractured porous media (F-AMS). 2015.

[53] O. Møyner and K.-A. Lie. A multiscale restriction-smoothed basis method for high contrast porous media represented on unstructured grids. J. Comput. Phys., 2015. submitted.

[54] O. Møyner and K.-A. Lie. A multiscale restriction-smoothed basis method for compressible black-oil models. SPE J., 2015. submitted.

[55] S. T. Hilden, O. Møyner, K.-A. Lie, and K. Bao. Multiscale simulation of polymer flooding with shear effects. 2015. submitted.

[56] M. A. Christie and M. J. Blunt. Tenth spe comparative solution project: A comparison of upscaling techniques. SPE Res. Eval. Eng., 4:308-317, 2001.

[57] G. Karypis and V. Kumar. A fast and high quality multilevel scheme for partitioning irregular graphs. SIAM J. Sci. Comput., 20(1):359-392, 1998. 
[58] Y. Wang, H. Hajibeygi, and H. A. Tchelepi. Algebraic multiscale solver for flow in heterogeneous porous media. J. Comput. Phys., 259(0):284-303, 2014.

[59] Y. Saad. Iterative Methods for Sparse Linear Systems. SIAM, Philadelphia, USA, 2003.

[60] The MATLAB Reservoir Simulation Toolbox (MRST), version 2015a. SINTEF Applied Mathematics, May 2015.

[61] K.-A. Lie. An Introduction to Reservoir Simulation Using MATLAB: User guide for the Matlab Reservoir Simulation Toolbox (MRST). SINTEF ICT, http://www.sintef.no/Projectweb/MRST/publications, 1 edition, May 2014.

[62] L. S. K. Fung and A. H. Dogru. Distributed unstructured grid infrastructure for complex reservoir simulation. In Europec/EAGE Conference and Exhibition, 2008.

[63] A. Kozlova, Z. Li, J. R. Natvig, S. Watanabe, Y. Zhou, K. Bratvedt, and S. H. Lee. A real-field multiscale black-oil reservoir simulator. In SPE Reservoir Simulation Symposium, Houston, Texas, USA, 2015. SPE 173226-MS.

[64] P. Vanek, J. Mandel, and M. Brezina. Algebraic multigrid by smoothed aggregation for second and fourth order elliptic problems. Computing, 56(3):179-196, 1996.

[65] P. Vanek. Acceleration of convergence of a two-level algorithm by smoothing transfer operator. Appl. Math., 37(4):265-274, 1992.

[66] H. Guillard and P. Vanek. An aggregation multigrid solver for convection-diffusion problems on unstructured meshes. Technical report, Center for Computational Mathematics, University of Colorado at Boulder, 1998.

[67] K.-A. Lie, S. Krogstad, I. S. Ligaarden, J. R. Natvig, H. M. Nilsen, and B. Skaflestad. Open-source matlab implementation of consistent discretisations on complex grids. Comput. Geosci., 16(2):297-322, 2012.

[68] S. Krogstad, K.-A. Lie, O. Møyner, H. M. Nilsen, X. Raynaud, and B. Skaflestad. MRST-AD - An open-source framework for rapid prototyping and evaluation of reservoir simulation problems. In SPE Reservoir Simulation Symposium, 2015.

[69] Fracture patterns in the Jandeira Formation. (NE Brazil). 3TU Datacentrum, http://data.3tu.nl/repository/uuid:be07fe95-417c-44e9-8c6a-d13f186abfbb, 2013. 